\title{
Das Kindeswohl und die Knochenmarkspende Minderjähriger aus verfassungsrechtlicher Perspektive
}

\author{
A. Katarina Weilert"
}

A. Problemaufriss

I. Medizinischer Hintergrund der Stammzellspende.

II. Rechtliche Regelung der Stammzellspende aus Knochenmark .......... 296

III. Gesetzliche Fallgruppen des $\mathbb{8}$ a TPG

B. Grundrechte und Minderjährige ....... 298

I. Differenzierung zwischen der Grundrechtsfähigkeit und Grundrechtsausübungsfähigkeit....

II. Eigene Grundrechtsausübung durch den Minderjährigen vs. stellvertretende Grundrechtsausübung ....... 299

1. Unterschiedliche Kriterien der Grundrechtsausübungsfähigkeit für unterschiedliche Bereiche.... 299

2. Fremdbestimmtes Wohl oder autonome Entscheidung des Minderjährigen?

3. Kriterium der Einsichtsfähigkeit für die Grundrechtsausübungsfähigkeit bzw. den Grundrechtsschutz im Medizinrecht........ 303

C. Verfassungsmäßigkeit von $\$ 8$ a TPG . . 304

I. Allgemeine Spendevoraussetzungen nach $\mathbb{} \$$ a TPG .................... 304

1. Das spendende Kind ............. 304

2. Die Spendesituation und Durchführung ....................... 306

3. Das Kindeswohl ............... 307

a) Definition des Kindeswohls nach $\mathbb{8} 8$ a TPG ............ 308

aa) Problematik der Definition des Kindeswohls ............ 308

bb) Mögliche Kindeswohlinterpretationen im Rahmen von $\$ 8$ a TPG (i.V.m. $\$ 1627$ BGB)

b) Verfassungsmäßigkeit des Kindeswohlkriteriums...... 310 aa) Verfassungsmäßigkeit der möglichen Kindeswohlinterpretationen ................ 310

bb) Zur Entscheidungsbefugnis der Eltern über das Vorliegen des „Kindeswohls“.... 313

cc) Zum Kindeswohlkriterium in Abhängigkeit von der Einsichtsfähigkeit ...

II. Verfassungsmäßigkeit der gesetzlichen Fallgruppen des $\mathbb{S} 8$ a TPG .... 315

1. Fallgruppe: Spendewillige Eltern und Baby ....

2. Fallgruppe: Spendewillige Eltern und Grundschulkind ........... 316

a) Aufklärung des Grundschulkindes.................... 316

b) Ablehnung der Spende durch das Grundschulkind ......... 316

aa) Menschenwürde ........... 318

bb) Recht auf körperliche Unversehrtheit und allgemeine Handlungsfreiheit .... 319

cc) Verfassungskonforme Auslegung des ,eingeschränkten Vetorechts" und Vorschlag einer Absicherung durch Kommissionsentscheidung

dd) Ausübungsfrist ............. 321

c) Bejahung der Spende durch das Grundschulkind ......... 322

3. Fallgruppe: Spendewillige Eltern und zustimmender Teenager ..... 322

4. Fallgruppe: Ablehnende Eltern und spendewilliger Teenager.... 323

a) Art. 6 Abs. 2 GG........... 323

b) Grundrechte des Teenagers. . 323

c) Fazit ...................... 325

* Dr. A. Katarina Weilert, LL.M. (London) ist Referentin am Arbeitsbereich Religion, Recht und Kultur der Forschungsstätte der Evangelischen Studiengemeinschaft e.V. in Heidelberg. Dieser Beitrag basiert auf einem Vortrag, den die Verfasserin am 15. November 2011 am Institut für Medizingeschichte und Wissenschaftsforschung der Universität zu Lübeck im Rahmen einer interdisziplinären Arbeitsgruppe zum Kindeswohl bei geschwisterlicher Gewebespende gehalten hat. In die vorliegende und vollständig überarbeitete Version sind Impulse aus anderen Beiträgen und Diskussionen dieser Tagung eingeflossen. 
D. Sicherung des Kindeswohls durch Ergänzungspflegschaft oder Kommission....

I. Ergänzungspflegschaft

( 1909 BGB)
II. Interdisziplinäre Kommission 327

E. Fazit ............................ 328

\section{A. Problemaufriss}

Die Stammzellspende ist zur Therapie von bestimmten Leukämieformen (Blutkrebs) sowie weiterer schwerer Krankheiten ${ }^{1}$ ein medizinisch gängiges Verfahren, das für manche Patienten die letzte Hoffnung auf Heilung und damit Überleben bedeutet, insbesondere wenn eine Chemo- oder Strahlentherapie erfolglos verlaufen ist. Leukämie ist eine Krebsart, die in etwa 50 \% der Fälle schon Kinder und Heranwachsende trifft. Bei der Stammzellspende durch Knochenmarktransplantation ${ }^{2}$ werden die gesunden Stammzellen des Spenders auf den kranken Patienten übertragen.

Nach $\int 8$ a Transplantationsgesetz (TPG) ist die Entnahme von Knochenmark bei Minderjährigen zugunsten eines Geschwisterkindes (sowie für die Eltern als Verwandte ersten Grades) unter bestimmten Voraussetzungen zulässig, selbst wenn das Spenderkind noch nicht einsichtsfähig (und damit noch nicht einwilligungsfähig) ${ }^{3}$ ist, sofern der gesetzliche Vertreter einwilligt. Der gesetzliche Vertreter, d.h. in aller Regel die Eltern ( $\mathbb{1 6 2 9}$ i.V.m. $\mathbb{S} 1626$ BGB), hat seine Entscheidung „zum Wohl des Kindes auszuüben“ ( $\mathbb{S} 8$ a Nr. 4 TPG i.V.m. $\$ 1627$ BGB). Die normalerweise für einen Heileingriff (im Gegensatz zum Wunscheingriff) erforderliche medizinische Indikation ist jedoch bei einer Spende nicht gegeben. ${ }^{4}$ Wie also ist dieses „Wohl“ als Leitmaßstab elterlicher Entscheidung zu verstehen, das die Legitimation für einen ärztlichen Eingriff gibt, der kein Heileingriff, sondern im strafrechtlichen Sinne eine

1 Die Stammzelltransplantation wird neben der Leukämie auch eingesetzt zur Behandlung einer aplastischen Anämie und Myelodysplasien, schwerer Immundefektsyndrome und einiger angeborener Stoffwechselerkrankungen (BÄK, Richtlinie für die allogene Knochenmarktransplantation mit nichtverwandten Spendern, Deutsches Ärzteblatt (91) 1994, A-761 (A-762)).

2 Auf die periphere Blutstammzellspende, deren Zulässigkeit sich nicht nach $\$ 8$ a TPG bemisst, und die juristisch besonders komplex ist und einer spezifischen spezialgesetzlichen Analyse bedarf, kann hier nicht näher eingegangen werden. Ein Vergleich aus medizinischer Perspektive findet sich bei S. Jansen, Die gerichtete allogene hämatopoetische Stammzellspende bei Kindern und Jugendlichen - Ein Vergleich von peripheren Stammzell- und Knochenmarkspendern, http://docserv.uni-duesseldorf.de/servlets/DerivateServlet/Derivate-15439/Druckfassung\%20PDF\%20A.pdf. Zur rechtlichen Einschätzung der Risiken: E. Deutsch/A. W. Bender/R. Eckstein/R. Zimmermann: Transfusionsrecht, 2. Aufl., Stuttgart 2007, Rn. 690; s. auch aus medizinischer Sicht K. Makita/K. Ohta/A. Mugitani/K. Hagihara/T. Ohta/ T. Yamane/M. Hino, Acute myelogenous leukemia in a donor after granulocyte colony-stimulating factor-primed peripheral blood stem cell harvest, Bone Marrow Transplantion 2004, S. $661 \mathrm{ff}$.; D. Stroncek/ Th. Shawker/ D. Follmann/ S.F. Leitman, G-CSF-induced spleen size changes in peripheral blood progenitor cell donors, Transfusion 2003, S. $609 \mathrm{ff}$.

3 BGHZ 29, 33 (37); B.-R. Kern, Die arzneimittelrechtliche Forschung an Minderjährigen und nicht einwilligungsfähigen Erwachsenen, in: B.-R. Kern/ H. Lilie, FS für G. Fischer, Frankfurt a.M. 2010, S. 146.

4 Vgl. U. Golbs, Das Vetorecht eines einwilligungsunfähigen Patienten, Baden-Baden 2006, S. 14, 16. 
Körperverletzung ${ }^{5}$ ist? Hinzu kommt, dass bei medizinisch nicht indizierten Eingriffen die Faustregel gilt

„je weniger ein Wunscheingriff indiziert ist, desto mehr hängt dessen Rechtfertigung von einer umfassenden Aufklärung und der sich darauf stützenden Einwilligung $a b " .6$

Diese Bedeutung der Einwilligung wirft besondere Probleme auf, wenn es um Minderjährige geht, deren Einwilligung stellvertretend von den Eltern wahrgenommen wird, die sowohl für das Wohl des Spenderkindes wie auch das des Empfängerkindes in Verantwortung stehen.

In diesem Beitrag soll erörtert werden, wie $\mathbb{8}$ a TPG verfassungsrechtlich zu bewerten ist.

\section{8 a Entnahme von Knochenmark bei minderjährigen Personen}

Die Entnahme von Knochenmark bei einer minderjährigen Person zum Zwecke der Übertragung ist abweichend von $\$ 8$ Abs. 1 Satz 1 Nr. 1 Buchstabe a und $b$ sowie Nr. 2 mit folgender Maßgabe zulässig:

1. Die Verwendung des Knochenmarks ist für Verwandte ersten Grades oder Geschwister der minderjährigen Person vorgesehen.

2. Die Übertragung des Knochenmarks auf den vorgesehenen Empfänger ist nach ärztlicher Beurteilung geeignet, bei ibm eine lebensbedrohende Krankheit zu beilen.

3. Ein geeigneter Spender nach $\mathbb{\int} 8$ Abs. 1 Satz $1 \mathrm{Nr} .1$ steht im Zeitpunkt der Entnahme des Knochenmarks nicht zur Verfügung.

4. Der gesetzliche Vertreter ist entsprechend $\mathbb{S} 8$ Abs. 2 aufgeklärt worden und hat in die Entnahme und die Verwendung des Knochenmarks eingewilligt. $\$ 1627$ des Bürgerlichen Gesetzbuchs ist anzuwenden. Die minderjährige Person ist durch einen Arzt entsprechend $\$ 8$ Abs. 2 aufzuklären, soweit dies im Hinblick auf ibr Alter und ihre geistige Reife möglich ist. Lehnt die minderjährige Person die beabsichtigte Entnahme oder Verwendung ab oder bringt sie dies in sonstiger Weise zum Ausdruck, so ist dies zu beachten.

5 Während die Meinungen für die strafrechtliche Beurteilung des Heileingriffs differieren (vgl. zum Streitstand B. Tag, Der Körperverletzungstatbestand im Spannungsfeld zwischen Patientenautonomie und Lex artis, Berlin/Heidelberg 2000, S. 13 ff.), wird der nicht indizierte Eingriff fast einhellig als tatbestandliche Körperverletzung beurteilt (vgl auch. Tag, a.a.O., S. 31).

6 S. Mennemeyer, Haftung ohne Grenzen?, in: Arbeitsgemeinschaft Rechtsanwälte im Medizinrecht e.V. (Hrsg.), Lifestyle-Medizin - von der medizinischen Indikation zum modischen Trend, Heidelberg (u.a.) 2012, S. 47 (50). 
5. Ist die minderjährige Person in der Lage, Wesen, Bedeutung und Tragweite der Entnahme zu erkennen und ibren Willen hiernach auszurichten, so ist auch ibre Einwilligung erforderlich.

Soll das Knochenmark der minderjährigen Person für Verwandte ersten Grades verwendet werden, hat der gesetzliche Vertreter dies dem Familiengericht unverzüglich anzuzeigen, um eine Entscheidung nach $\$ 1629$ Abs. 2 Satz 3 in Verbindung mit $\$ 1796$ des Bürgerlichen Gesetzbuchs herbeizufübren.

\section{Medizinischer Hintergrund der Stammzellspende}

Für das Spenderkind verläuft die Knochenmarkentnahme unter Vollnarkose. Dabei werden einem Klein- und Schulkind zwischen 140 bis $820 \mathrm{ml}$ Knochenmarkblut am rechten und linken Beckenkamm entnommen. ${ }^{7}$ Das Entnahmevolumen richtet sich dabei unter anderem nach dem Körpergewicht des Spenders. Für die Entnahme an den Beckenkämmen werden multiple Punktionen - meist sternförmig und mit mehreren Hautschnitten verbunden - gesetzt. Teilweise ist es notwendig, den Blutverlust des Spenderkindes auszugleichen, entweder über kolloidale Lösungen oder über autologe Erythrozyten, die dem Kind zuvor durch Eigenspende entnommen wurden. Für den Eingriff und die spätere Überwachung müssen die Kinder im Durchschnitt 3-5 Tage (Streuung 2-8 Tage) im Krankenhaus verbleiben. Das Empfängerkind muss vor der Transplantation einer gravierenden Behandlung, der sog. „Konditionierung", unterzogen werden, bei der sein erkranktes Knochenmark zerstört wird. Überdies muss sein Immunsystem supprimiert werden, damit es nicht zu AbstoBungsreaktionen kommt. Beides wird im Wege einer Chemotherapie, oft in Kombination mit einer Ganzkörperbestrahlung, erzielt. Für den Empfänger sind mit der Konditionierung und Knochenmarktransplantation hohe Risiken verbunden, vor allem ein Infektionsrisiko, das sog. „graft versus host disease“ (GvHD), ${ }^{8}$ Spätfolgen der Therapie und auch ein Wiederaufkeimen des Krebses (Tumorrezidive). Die Erfolgschancen hängen u.a. von der Art der Leukämie und dem Fortschritt des Krankheitsverlaufes ab. ${ }^{9}$

Als Spender kommen nur diejenigen Menschen in Frage, die über fast identische Gewebemerkmale verfügen wie der Empfänger. Diese Übereinstimmung zwischen zwei nicht miteinander verwandten Menschen ist so selten, dass nur durch Knochenmarkspender-Dateien überhaupt eine Chance besteht, dass ein geeigneter Spen-

7 Vgl. für den medizinischen Ablauf die Spenderinformation der Österreichischen Knochenmarkspendezentrale, wiedergegeben bei A. Schmidt-Recla, Kontraindikation und Kindeswohl. Die „zulässige“ Knochenmarkspende durch Kinder, GesR 2009, S. 566 (basierend auf http://www.stammzellspende.at/ main.asp?content=spende-grundsaetze.asp).

8 Transplantat „erkennt“ den Empfänger als fremd und greift Organe wie Leber, Darm und Haut des Empfängers an (vgl. http://www.krebsgesellschaft.de/leukaemie_therapie_knochenmark_blutstammzelltransplantation,39095.html).

9 http://www.leukaemie-online.de/index.php?option=com_content\&view=article\&id=19\&Itemid=28. 
der gefunden werden kann. Auch innerhalb einer Familie besteht fast nie ausreichende Identität der Gewebemerkmale zwischen Eltern und ihren Kindern, da Kinder nur die Hälfte des Chromosomensatzes von ihrem jeweiligen Elternteil erben. Statistisch gesehen gibt es eine Wahrscheinlichkeit der Übereinstimmung zwischen elterlichen (biologischen) HLA-Haplotypen und denjenigen ihrer Kinder im Verhältnis von 1:200. ${ }^{10}$ Geschwister des erkrankten Kindes kommen dagegen mit einer statistischen Wahrscheinlichkeit von $25 \%$ als Spender in Frage. ${ }^{11}$

Dies wirft die Frage nach einer gesetzlichen Regelung für die Knochenmarkspende Minderjähriger auf.

\section{Rechtliche Regelung der Stammzellspende aus Knochenmark}

In Deutschland war die Knochenmarkspende lange Zeit ungeregelt, sowohl für Erwachsene als auch für Kinder. $\ 8$ a TPG, der die Knochenmarkspende von Minderjährigen auf eine gesetzliche Grundlage stellt, wurde erst im Jahre 2007 durch das Gewebegesetz in das Transplantationsgesetz aufgenommen. Obwohl die Einfügung von $\mathbb{\$} 8$ a TPG mit den Grundsätzen der Organ- und Gewebespende, nämlich der Einwilligungsfähigkeit des Spenders und der Freiwilligkeit der Spenderentscheidung, bricht, ist die gesetzliche Legitimierung der Kinderspende relativ geräuschlos durch den Bundestag gewunken worden. ${ }^{12}$ Die EU-RL, ${ }^{13}$ die durch das Gewebegesetz umgesetzt wurde, hatte die Regelung der Knochenmarkspende von Minderjährigen nicht explizit verlangt. Die EU-RL sieht allerdings vor, dass die Knochenmarkspende bestimmten Anforderungen genügen muss, so dass eine gesetzliche Regelung notwendig wurde. ${ }^{14} \mathrm{Da} \ 8$ TPG bestimmt, dass die Spender volljährig und einwilligungsfähig sein müssen und eine Spende nur freiwillig erfolgen darf, bedeutete die Aufnahme der Knochenmarkspende in das TPG, dass - ohne die Sonderregelung in $\$ 8$ a TPG - eine Kinderspende fortan nicht mehr hätte stattfinden dürfen. Offenkundig wollte man der gängigen Praxis der Kinderspenden ${ }^{15}$ aber keinen Riegel vorschieben und stellte diese daher mit $\mathbb{8} 8$ a TPG auf eine rechtliche Grundlage.

10 In seltenen Ausnahmen gibt es eine $50 \%$ ige Wahrscheinlichkeit, wenn bei beiden Elternteilen ein identischer Haplotyp vorliegt. R.F. Schipper/ J. D'Amaro/ M. Oudshoorn, The probability of finding a suitable related donor for bone marrow transplantation in extended families, Blood 1996, S. $800 \mathrm{ff}$.

11 J.A. Hansen, in: L. Frohnmayer/D. Frohnmayer, Fanconi Anemia, A Handbook for Families and Their Physicians, 3. Aufl. 2000, Fanconi Anemia Research Fund, Inc., Eugene, Oregon, S. 74 ff.

12 Vgl. H.-G. Gerdts, $\mathbb{S} 8$ a TPG und der Grundsatz des Verbots fremdnütziger medizinischer Eingriffe in die körperliche Integrität ohne eigene Zustimmung, WDR Hörfunk 100,1 Ausstrahlungsdatum: 17.10.2011.

13 Richtlinie 2004/23/EG des Europäischen Parlaments und des Rates vom 31. März 2004 zur Festlegung von Qualitäts- und Sicherheitsstandards für die Spende, Beschaffung, Testung, Verarbeitung, Konservierung, Lagerung und Verteilung von menschlichen Geweben und Zellen (ABl. EU Nr. L 102 S. 48).

14 Vgl. insbesondere Art. 12 ff. RL 2004/23/EG.

15 Man geht von jährlich ca. 100 Knochenmarkspenden durch Minderjährige vor Erlass des $\int 8$ a TPG aus (vgl. die Anhörung des Vertreters des Zentralen Knochenmarkspenderregisters Deutschland vor dem Bundestagsausschuss für Gesundheit am 7. März 2007, 44. Sitzung des Bundestagsausschusses für Gesundheit, Protokoll Nr. 16/44, S. 10). 
Zur Erlaubnis einer Spende durch nichteinwilligungsfähige Erwachsene konnte man sich allerdings nicht durchringen, vielmehr fürchtete man einen Tabubruch. ${ }^{16} \mathrm{Im}$ merhin liegt einer der Gründe für Deutschlands ablehnende Haltung gegenüber dem „Übereinkommen zum Schutz der Menschenrechte und der Menschenwürde im Hinblick auf die Anwendung von Biologie und Medizin: Übereinkommen über Menschenrechte und Biomedizin“ (oft als Bioethik- oder Biomedizinkonvention bezeichnet) gerade in dem Argument, dass die nicht einwilligungsfähigen Menschen nicht hinreichend geschützt sind. ${ }^{17}$ Art. 20 Abs. 2 der Konvention gestattet unter bestimmten Bedingungen, dass die Vertragsstaaten Regelungen etablieren, nach denen auch einwilligungsunfähigen Menschen regenerierbares Gewebe entnommen werden darf. Allerdings erlaubt die Konvention ausdrücklich, dass Staaten ein höheres Schutzniveau verankern, so dass sich hieraus selbst für Konventionsstaaten keine Pflicht zu einer solchen Ermächtigung ableiten lässt (vgl. Art. 27 der Konvention). Verboten ist eine Gewebeentnahme bei Einwilligungsunfähigen jedenfalls, wenn der Spender diese ablehnt (Art. 20 Abs. 2 lit. v der Konvention).

$\$ 8$ a TPG betrifft im Kern die Frage nach der Selbstbestimmung und dem Grundrechtsschutz Minderjähriger im Medizinrecht. Welche Voraussetzungen müssen aber gegeben sein, damit man überhaupt von einer selbstbestimmten Entscheidung sprechen kann? Bei Organ- und Gewebespenden innerhalb einer Familie gibt es aufgrund der Nähebeziehung nicht nur eine hohe Motivation für den Spender, sondern es kann auch einen spürbaren Erwartungsdruck seitens der Familie, im Falle der kindlichen Knochenmarkspende besonders der Eltern, geben. ${ }^{18}$

Zunächst soll gezeigt werden, welche Fallgruppen mit jeweils unterschiedlichen Anforderungen $\mathbb{8}$ a TPG für die Knochenmarkspende Minderjähriger bildet.

\section{Gesetzliche Fallgruppen des § 8 a TPG}

Der $\ 8$ a TPG regelt die verschiedenen Fallkonstellationen einer Knochenmarkspende minderjähriger Personen in Abhängigkeit ihrer Einsichtsfähigkeit (und damit Ein-

16 Schmidt-Recla, Kontraindikation, (Fn. 7), S. 568; U. Walter, in: A. Spickhoff (Hrsg.) Medizinrecht, München 2011, $\mathbb{S} 8$ a TPG Rn. 1.

17 Vgl. zur Kritik http://www.bioethik-konvention.de/; BT-Drs. 16/5443, S. 51 („Vor dem Hintergrund, dass die Bundesrepublik Deutschland das 'Übereinkommen zum Schutz der Menschenrechte und der Menschenwürde im Hinblick auf die Anwendung von Biologie und Medizin: Übereinkommen über Menschenrechte und Biomedizin (Bioethikkonvention)' des Europarates nicht unterzeichnet hat, da sie die dort vorgesehenen Eingriffe bei Nichteinwilligungsfähigen als zu weitgehend betrachtet, sollte sie diese Gesichtspunkte auch bei ihrer nationalen Gesetzgebung berücksichtigen.").

18 W. Roell, Die Geschwister krebskranker Kinder, Bern (u.a.) 1996, S. 307: „In einzelnen Fällen dieser Untersuchung wurden die Spender denn auch offen mit dem Hinweis konfrontiert, dass das Geschwister sonst sterben müsse; unter diesem Druck konnten sie gar nicht anders als der Spende zuzustimmen. “; Vgl. auch B. Fateh-Moghadam/U. Schroth/Ch. Gross/Th. Gutmann, Die Praxis der Lebendspendekommissionen - Eine empirische Untersuchung zur Implementierung prozeduraler Modelle der Absicherung von Autonomiebedingungen im Transplantationswesen, MedR 2004, S. 19 ff. 
willigungsfähigkeit) ${ }^{19}$ und in Abhängigkeit der Einwilligung der Eltern. Damit bestehen Parallelen zu $\mathbb{4} 40$ Abs. 4 Nr. 3 Arzneimittelgesetz (AMG) im Hinblick auf die Zulässigkeit klinischer Arzneimittelprüfungen. Die Einwilligung der Eltern ersetzt die Einwilligung des Spenderkindes ( $\$ 8$ a Nr. 4 TPG). Je nach Einsichtsfähigkeit des Minderjährigen wird seinem Willen unterschiedliches Gewicht beigemessen. $\mathbb{8} 8 \mathrm{a}$ TPG unterscheidet zwischen drei Stufen der Einsichtsfähigkeit des Minderjährigen und differenziert danach die Beachtlichkeit seiner Willensäußerung. Zur Vereinfachung soll im Folgenden das „Baby“ für den nicht einsichtsfähigen Minderjährigen stehen, das „Grundschulkind“ für den teileinsichtsfähigen Minderjährigen und der „Teenager" für den einsichtsfähigen Minderjährigen. Darüber hinaus differenziert $\$ 8$ a TPG danach, ob die gesetzlichen Vertreter (in der Regel die Eltern) die Spende befürworten („Einwilligung“) oder ablehnen („keine Einwilligung“).

Nun lassen sich zwischen den verschiedenen Stufen der Minderjährigkeit und der Zustimmung/Ablehnung der Eltern alle Variationen bilden: Beim Baby entscheiden immer die Eltern über die Spende, da das Baby keinen eigenen Willen im Hinblick auf die Spende bilden kann. Beim (teileinsichtsfähigen) Grundschulkind dürfen die Eltern auch so gut wie allein entscheiden, jedoch ist seine Ablehnung „zu beachten“. Beim Teenager ist sowohl die Einwilligung der Eltern als auch die des Minderjährigen erforderlich. Problematisch ist nun insbesondere die Vornahme der Spende in den Fallkonstellationen „Baby und spendewillige Eltern“ sowie „ablehnendes Grundschulkind und spendewillige Eltern“. Ebenso ist das Spendeverbot für den zustimmenden Teenager zu hinterfragen, dessen Eltern ihre Einwilligung nicht erteilen.

\section{B. Grundrechte und Minderjährige}

Bevor im Einzelnen erörtert werden soll, ob $\mathbb{8}$ a TPG in Grundrechte des Minderjährigen eingreift bzw. diese nicht ausreichend schützt, ist zu bestimmen, inwieweit sich Minderjährige auf Grundrechte berufen können. ${ }^{20}$

19 S. Fn. 3.

20 Im Einzelnen ist hier sowohl terminologisch als auch inhaltlich fast alles umstritten (vgl. für einen komprimierten Überblick I. v. Münch/Ph. Kunig, in: I. v. Münch/P. Kunig (Hrsg.), Grundgesetzkommentar, Bd. 1, 6. Aufl., München 2012, Vorb. Rn. 31; ausführlich bei W. Roth, Die Grundrechte Minderjähriger im Spannungsfeld selbständiger Grundrechtsausübung, elterlichen Erziehungsrechts und staatlicher Grundrechtsbindung, Berlin 2003; M. Brüser, Die Bedeutung der Grundrechte im Kindesalter für das „Elternrecht“, Frankfurt a.M. 2010). 


\section{Differenzierung zwischen der Grundrechtsfähigkeit und Grundrechtsausübungsfähigkeit ${ }^{21}$}

Die Grundrechtsfähigkeit oder auch Grundrechtsträgerschaft/Grundrechtsberechtigung kommt im Hinblick auf die meisten Grundrechte jedem Menschen unabhängig von seinem Alter, ${ }^{22}$ also auch dem Säugling, zu. ${ }^{23}$ Für die in Bezug auf das Spenderkind in Frage stehenden Grundrechte der allgemeinen Handlungsfreiheit (Art. 2 Abs. 1 GG), des allgemeinen Persönlichkeitsrechts (Art. 2 Abs.1 i.V.m. Art. 1 Abs. 1 GG) und des Rechts auf körperliche Unversehrtheit (Art. 2 Abs. 2 GG) ist die Grundrechtsfähigkeit Minderjähriger allgemein anerkannt. Im Hinblick auf die Verfassungsmäßigkeit von $\mathbb{\int} 8$ a TPG ist zentral, ob und inwieweit der Minderjährige als Grundrechtsträger seine Grundrechte im Alltag ohne fremde Hilfe ausüben kann. Diese Fähigkeit soll hier als „Grundrechtsausübungsfähigkeit“24 (oder auch „Grundrechtsreife“25 bzw. „Grundrechtseigenständigkeit“) ${ }^{26}$ bezeichnet werden. Sie markiert die Grenze zwischen dem Elternrecht und eigenverantwortlichem kindlichen Handeln.

\section{Eigene Grundrechtsausübung durch den Minderjährigen vs. stellvertretende Grundrechtsausübung}

1. Unterschiedliche Kriterien der Grundrechtsausübungsfähigkeit für unterschiedliche Bereiche

Anders als das Zivilrecht normiert das Grundgesetz keine allgemeinen starren Altersgrenzen für die Grundrechtsausübung bzw. Vertretung durch die Eltern. Da die Ausübung der verschiedenen Grundrechte sehr unterschiedliche geistige Reifezustände voraussetzt, kann es keine einheitliche Grundrechtsausübungsfähigkeit geben. Einfachgesetzliche Altersangaben können dabei die Grundrechtsausübung nur einschränken, soweit sie verfassungsmäßig sind.

Die Verfassungsmäßigkeit kann man für diejenigen zivilrechtlichen Regelungen annehmen, die rechtsgeschäftliches Handeln betreffen. ${ }^{27}$ Hier ist in verhältnismäßiger

21 Die Grundrechtsmündigkeit wird hier verstanden als ein Fachbegriff für die prozessuale Durchsetzung der Grundrechte. Sie spielt an dieser Stelle keine zentrale Rolle und kann daher hier außen vorgelassen werden. Näher hierzu H. Dreier in: H. Dreier (Hrsg.), Grundgesetz-Kommentar, Bd. I, 2. Aufl., Tübingen 2004, Vorb. Rn. 114 mit weiteren Nachweisen; vgl. zu „Mündigkeitskonzepten im Recht“ M. Zitelmann, Kindeswohl und Kindeswille im Spannungsfeld von Pädagogik und Recht, Münster 2001, S. 73.

22 Vgl. aber Art. 38 Abs. 2 GG und Art. 12 a Abs. 1 GG.

23 Vgl. M. Sachs, in M. Sachs (Hrsg.), Grundgesetz-Kommentar, 6. Aufl., München 2011, Vor Art. 1 Rn. 70 ff.; die Fragen der „Ränder“ der Grundrechtsträgerschaft, d.h. am Lebensanfang (Embryo) und Lebensende können hier außen vor gelassen werden.

24 Auch die Terminologie der Grundrechtsausübungsfähigkeit wird unterschiedlich gebraucht. Sie wird bereits verwendet bei K. Stern, Staatsrecht, Bd. III/1, München 1988, S. 1068.

25 Vgl. Roth, Die Grundrechte Minderjähriger (Fn. 20), S. 46.

26 Brüser, Grundrechte im Kindesalter (Fn. 20), S. 70.

27 Stern, Staatsrecht (Fn. 24), S. 1069. 
Weise einerseits der Einsichtsfähigkeit Minderjähriger, ihrem Interesse an der Teilnahme am Rechtsverkehr, dem Schutz der Minderjährigen und zugleich der notwendigen Rechtssicherheit Rechnung getragen worden.

Für die Frage der Grundrechtsausübung im nicht rechtsgeschäftlichen Verkehr sind die Determinanten des Schutzes, der Autonomie und Rechtssicherheit wieder anders $\mathrm{zu}$ bestimmen. ${ }^{28}$ Die Situation einer Knochenmarkspende unterscheidet sich in grundsätzlicher Weise von rechtsgeschäftlichem Handeln. Während sich beim Abschluss eines Kaufvertrages Käufer und Verkäufer nicht einmal notwendigerweise begegnen müssen, gibt es bei einem ärztlichen Eingriff in der Regel aufklärende persönliche Vorgespräche. Die Anforderungen an Rechtssicherheit und Reibungslosigkeit des rechtsgeschäftlichen Handelns können daher nicht unbesehen auf das Medizinrecht übertragen werden.

\section{Fremdbestimmtes Wohl oder autonome Entscheidung des Minderjährigen?}

Die Minderjährigen stehen damit in einer Spannung zwischen einem fremdbestimmten Wobl und der autonomen Grundrechtsausübung. Es streiten also gegeneinander das Prinzip der Fürsorge, primär durch die Eltern und sekundär durch den Staat nach Art. 6 Abs. 2 GG, und das den Grundrechten als Freiheitsrechten innewohnende Prinzip der Autonomie. Die Spannung zwischen fremdbestimmtem Wohl und autonomer Grundrechtsausübung besteht dabei in vielen Lebensbereichen. Wie diese Spannung jeweils aufgelöst wird, ist in hohem Maße von einem gesellschaftlichen Konsens und der kulturellen Prägung einer Gesellschaft abhängig. Dabei unterliegt das, was als angemessene Lösung empfunden wird, einem stetigen Wandel.

Für den Bereich der kindlichen Knochenmarkspende sind zwei Determinanten von besonderem Gewicht: Erstens die grundsätzliche Einstellung einer Gesellschaft den Kindern und ihrer Einsichtsfähigkeit gegenüber und zweitens der ethische Minimalkonsens in Bezug auf die Gabe körpereigener Substanzen. Für letztere gilt das Prinzip der Freiwilligkeit.

Im Hinblick auf die Einstellung Kindern gegenüber ist ein gewaltiger gesellschaftlicher Wandel seit Anfang des 20. Jahrhunderts zu verzeichnen, der hier nur ange-

28 Zur Unterscheidung von Behandlungsvertrag und ärztlicher Heilmaßnahme Golbs, Das Vetorecht, (Fn. 4), S. 49 ff.; B.-R. Kern, Fremdbestimmung bei der Einwilligung in ärztliche Eingriffe, NJW 1994, S. 753.; Zur Unterscheidung zwischen einer rechtsgeschäftlichen Willenserklärung und einer tatsächlichen Willenserklärung am Beispiel des Schwangerschaftsabbruchs bei Minderjährigen: Hier wird zwischen der den Behandlungsvertrag begründenden Willenserklärung (Geschäftsfähigkeitsregeln nach BGB) und der Einwilligung zum Schwangerschaftsabbruch (Einwilligungsfähigkeit nach Strafrecht) unterschieden: P. Moritz, Bedeutung des Elternvotums für den Abbruch der Schwangerschaft Minderjähriger, ZfJ (Zentralblatt für Jugendrecht) 1999, S. 92 (S. 99, Zusammenfassung Nr. 1); $H$. Kaup, Der Schwangerschaftsabbruch aus verfassungsrechtlicher Sicht, Frankfurt am Main 1991, S. 183; wohl anderer Auffassung D. Olzen, in: D. Schwab (Hrsg.), Münchener Kommentar zum Bürgerlichen Gesetzbuch, Bd. 8., 5. Aufl., München 2010, \$1666 Rn. 76 ff. Zutreffend weist Olzen darauf hin, dass beim Schwangerschaftsabbruch die spezielle Situation vorliegt, dass die Entscheidung einen dritten Menschen (das ungeborene Kind) betrifft (Rn. 81). 
deutet werden kann und der sich erst nach und nach im Recht widerspiegelte. Vielfältige Faktoren haben diese veränderte gesellschaftliche Einstellung zu Kindern, die mehr und mehr als Individuen mit zu berücksichtigenden eigenen Interessen und Bedürfnissen wahrgenommen wurden, beeinflusst. Gründe für die lange Zeit unterentwickelte Berücksichtigung des Kindeswillens lagen in Traditionen, einem Desinteresse dem Kind gegenüber und der Vormachtstellung von Eltern gegenüber ihren Kindern. Mit der Einführung des neuen Scheidungsrechts in den 70er Jahren, in der vor allem das Schuldprinzip vom Zerrüttungsprinzip ${ }^{29}$ abgelöst wurde, bedurfte auch die Sorgerechtsentscheidung neuer Kategorien. ${ }^{30}$ Während früher das Sorgerecht automatisch der Mutter oder dem Elternteil zugesprochen wurde, welches nicht am Scheitern der Ehe schuld war, rückte nun das Kindeswohl in den Mittelpunkt.

Das Familienrecht war insgesamt Vorreiter für die Beachtung des Kindeswillens und Kindeswohls im Recht. Schon 1968 stellte das BVerfG in einem Beschluss (zur Adoption) klar:

„Das Kind ist ein Wesen mit eigener Menschenwürde und dem eigenen Recht auf Entfaltung seiner Persönlichkeit im Sinne der Art. 1 Abs. 1 und Art. 2 Abs. 1 GG. Eine Verfassung, welche die Würde des Menschen in den Mittelpunkt ihres Wertsystems stellt, kann bei der Ordnung zwischenmenschlicher Beziehungen grundsätzlich niemandem Rechte an der Person eines anderen einräumen, die nicht zugleich pflichtgebunden sind und die Menschenwürde des anderen respektieren. Die Anerkennung der Elternverantwortung und der damit verbundenen Rechte findet daher ibre Rechtfertigung darin, daß das Kind des Schutzes und der Hilfe bedarf, um sich zu einer eigenverantwortlichen Persönlichkeit innerhalb der sozialen Gemeinschaft zu entwickeln, wie sie dem Menschenbilde des Grundgesetzes entspricht (vgl. BVerfGE 7, 198 [205]; s. a. die Erklärung der Vereinten Nationen über die Rechte des Kindes vom 20. November 1959 [Yearbook of the United Nations, 1959, S. 198]). Hierüber muß der Staat wachen und notfalls das Kind, das sich noch nicht selbst zu schützen vermag, davor bewahren, daß seine Entwicklung durch einen Mißbrauch der elterlichen Rechte oder eine Vernachlässigung Schaden leidet. " 31

Erst Ende der 70er Jahre wurde der schon vom BVerfG formulierte Gedanke in ein neues Erziehungsleitbild gegossen, dass nicht mehr auf einem autoritären Gehorsamsgrundsatz basierte, sondern der Entwicklung des Kindes als heranwachsendem, zunehmend selbstständigem und verantwortungsbewusstem Menschen Rechnung

30 Zitelmann, Kindeswohl und Kindeswille (Fn. 21), S. 114.

31 BVerfGE 24, 119 (144). 
trug. ${ }^{32} 1998$ wurden die prozessualen Rechte des Kindes mit $\ 50$ FGG (heute $\mathbb{1} 158$ FamFG) durch die Möglichkeit der Verfahrenspflegschaft verstärkt, wonach für das Kind ein Pfleger (heute Verfahrensbeistand) zu bestellen war, wenn dies zur Wahrnehmung seiner Interessen erforderlich schien. ${ }^{33}$ Die Erforderlichkeit war vor allem angezeigt, wenn ein Interessengegensatz zwischen Kind und gesetzlichem Vertreter bestand. Erst diese Entwicklungen im Familienrecht führten dazu, dass die Grundrechtsträgerschaft des Kindes wahrgenommen und dass das Elterngrundrecht entsprechend interpretiert bzw. einfachgesetzlich angepasst wurde.

Wenn $\ 50$ FGG / $\$ 158$ FamFG vom „Interesse“ des Kindes spricht, so muss geklärt werden, ob damit das - objektive - Kindeswohl oder der - individuelle - Kindeswille gemeint ist. Es sprechen gute Gründe dafür, das Interesse sowohl als Wohl als auch als Willen des Kindes zu interpretieren. ${ }^{34}$ Das Zusammenspiel beider im Rahmen von \$158 FamFG ist jedoch nicht gänzlich klar. Bei einem offensichtlichen Widerspruch zwischen subjektivem Kindeswillen und objektivem Kindeswohl ist letzteres ausschlaggebend. ${ }^{35}$

Allerdings kann dies nur gelten, solange der kindliche Wille aufgrund nicht voller Einsichtsfähigkeit als defizitär angesehen wird. Zum einen ist das Kindeswohl teils nicht objektiv bestimmbar, zum anderen werden auch Erwachsene nicht vor sich selbst geschützt, selbst wenn objektiv eine andere Entscheidung ihrem Wohl besser gedient hätte. Das Kindeswohl hat eine „Ersatzfunktion für den Willen des Kindes, solange dieser rechtlich nicht anerkannt wird. " ${ }^{36}$ Das Kindeswohl rückt also an die Stelle des Kindeswillens, solange und soweit dem Unmündigen ein beachtlicher eigener Wille noch nicht zuerkannt wird. Das Kindeswohl zielt grundrechtsdogmatisch eher in Richtung des Schutzes durch den Staat (im Falle eines Interessengegensatzes zu den Eltern), der Kindeswille auf eine Stärkung der kindlichen Interessen. Das Kindeswobl deutet also auf das Kind als Grundrechtsträger im Sinne eines Grund-

32 Zitelmann, Kindeswohl und Kindeswille (Fn. 21), S. 74; \ 1626 Abs. 2 BGB: „Bei der Pflege und Erziehung berücksichtigen die Eltern die wachsende Fähigkeit und das wachsende Bedürfnis des Kindes zu selbständigem verantwortungsbewusstem Handeln. Sie besprechen mit dem Kind, soweit es nach dessen Entwicklungsstand angezeigt ist, Fragen der elterlichen Sorge und streben Einvernehmen an."

33 Ein Vorläufer ist der „Curator“ im preußischen Recht, der bei einer Interessenskollision von Kindern und Eltern hinzugezogen wurde, vgl. Zitelmann, Kindeswohl und Kindeswille (Fn. 21), S. 150.

34 H. Engelhardt, in: H. Engelhardt/W. Sternal (Hrsg.), FamFG Kommentar, 17. Aufl., München 2011, \$158 Rn. 20: Kindeswille als „ein essentielles Element des vom Gericht zu ermittelnden Kindeswohls“; R. Kemper, in: Saenger (Hrsg.) Zivilprozessordnung/ FamFG/ Europoäisches Verfahrensrecht, Handkommentar, 4. Aufl., Baden-Baden 2011, \ 158 Rn. 16, versteht das Interesse in erster Linie als Kindeswille bei gleichzeitiger Verpflichtung auf das Wohl des Kindes. Eine tendenziell stärkere Betonung des Kindeswohls findet sich bei M.Völker/M. Clausius, in: R. Kemper/K.Schreiber (Hrsg.), Familien-

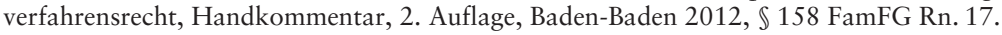

35 Engeldhardt (Fn. 34) $\mathbb{1 5 8}$ Rn. 20.

36 J. Münder/E. Kühn, Mögliche Aufgaben empirischer Wissenschaften im Familienrecht, verdeutlicht am Beispiel des Kindeswohls, in N. Horn/R. Tietz (Hrsg.), JUS-Didaktik, Heft 3, Sozialwissenschaften im Studium des Rechts, Bd. 1 Zivil—und Wirtschaftsrecht, München 1977, S. 99. 
rechtsgeschützten (Schutzpflicht), das Kindesinteresse auf die Ausübung des Grundrechts als Freiheitsrecht durch das Kind.

Auch heute noch wird der Kindeswille im Recht beschränkt, entweder zugunsten allgemeiner Interessen (z.B. der „Sicherheit des Rechtsverkehrs“) oder zugunsten des Kindeswohls selbst (etwa bei überfordernden Entscheidungssituationen). ${ }^{37}$ Inwieweit dies verfassungsgemäß ist, muss jeweils sorgfältig geprüft werden und wird wohl auch im Rahmen sich wandelnder sozialer Normen ${ }^{38}$ und wissenschaftlicher Erkenntnisse immer wieder neu zu verhandeln sein.

\section{Kriterium der Einsichtsfähigkeit für die Grundrechtsausübungsfähigkeit bzw. den Grundrechtsschutz im Medizinrecht}

Im Bereich des Medizinrechts, in dem nicht die „Sicherheit des rechtsgeschäftlichen Verkehrs" zu schützen ist, eignet sich als Kriterium der Abgrenzung zwischen eigener und stellvertretender Grundrechtsausübung das Kriterium der Einsichtsfähigkeit, ${ }^{39}$ sofern die Ausübung des jeweiligen Grundrechts eine bestimmte geistige Kapazität erfordert. Einsichtsfähigkeit setzt dabei sowohl eine bestimmte intellektuelle Fähigkeit (Begreifen der Dinge und Reflexionsvermögen) voraus als auch die soziale Befähigung, in bestimmtem Maße Verantwortung für sich und andere übernehmen zu können. ${ }^{40}$ Ebenso muss das Kind bei einer Grundrechtsausübung, die für die Zukunft Implikationen hat, in der Lage sein, die Zukunftsdimension zu erfassen. Hier gilt es zu bedenken, dass Kinder viel mehr als Erwachsene im „Jetzt“ leben und sich zeitliche Räume aufgrund ihrer eigenen kurzen Lebenszeit noch nicht in gleicher Weise wie Erwachsene vergegenwärtigen können. Damit ist die „Einsichtsfähigkeit“ eine sehr komplexe Größe und die Anforderungen, die an sie zu stellen sind, müssen unterschiedlich, entsprechend der Tragweite der grundrechtsrelevanten Entscheidung, ausfallen. Der Grad an erforderlicher Einsichtsfähigkeit variiert damit nicht nur zwischen den verschiedenen Grundrechten, ${ }^{41}$ sondern auch im Hinblick auf die konkrete grundrechtsrelevante Entscheidung. Dies wiederum bedeutet ein hohes Maß an Rechtsunsicherheit, zum einen im Hinblick auf die Definition des anzulegenden Maßstabes und zum anderen im Hinblick auf die Frage, ob der Minderjährige diesem Maßstab gerecht wird.

38 Für die Relevanz sozialer Normen bei der Bestimmung des Elternrechts s. R. Gröschner, in: H. Dreier (Hrsg.), Grundgesetz-Kommentar, Bd. I, 2. Aufl., Tübingen 2004, Art. 6 Rn. 121; D. Coester-Waltjen, in: I. v. Münch/P. Kunig (Hrsg.), Grundgesetz-Kommentar, Bd. 1, 6. Aufl., München 2012, Art. 6 Rn. 81.

39 Vgl. zum Kriterium der Einsichtsfähigkeit Dreier (Fn. 21), Vorb. Rn. 113; Stern, Staatsrecht (Fn. 24), S. 1068 f.; für die Einwilligung zum Schwangerschaftsabbruch bei Minderjährigen Kaup, Der Schwangerschaftsabbruch aus verfassungsrechtlicher Sicht (Fn. 28), S. 184. Anderer Ansicht (für eine starre Altersgrenze) I. v. Münch, Staatsrecht II, 5. Aufl., Stuttgart 2002, Rn. 104 ff.; A. Bleckmann, Staatrecht II - Die Grundrechte, 4. Aufl., Köln u.a. 1997, $\$ 17$ III 1.

41 Stern, Staatsrecht (Fn. 24), S. 1068 f. 
Eine Auslegung der Ausübungsfähigkeit der Grundrechte nach dem Maße der Einsichts- und damit Einwilligungsfähigkeit steht auch in Übereinstimmung mit internationalem Recht. Art. 12 der UN-Kinderrechtskonvention sieht vor, dass die Meinung des Kindes entsprechend seinem Alter und seiner Reife zu berücksichtigen ist. Für gerichtliche Verfahren bestimmt die Kinderrechtskonvention eine Anhörung des Kindes. Nach Art. 4 des Europäischen Übereinkommens über die Ausübung von Kinderrechten können Kinder einen besonderen Vertreter in Verfahren vor Justizbehörden beantragen, wenn ein Interessenskonflikt zwischen Eltern und Kindern besteht. Dieses Recht kann allerdings auf Kinder beschränkt werden, „die nach innerstaatlichem Recht als hinreichend verständig angesehen werden."

Im Folgenden ist nun $\mathbb{} 8$ a TPG im Lichte der Grundrechte des Minderjährigen zu bewerten.

\section{Verfassungsmäßigkeit von $\S 8$ a TPG}

Vorschriften aus Bundesgesetzen sind nichtig, wenn sie mit dem Grundgesetz unvereinbar sind und eine verfassungskonforme Auslegung nicht möglich ist. ${ }^{42}$

\section{Allgemeine Spendevoraussetzungen nach § 8 a TPG}

Die Tatbestandsvoraussetzungen des $\mathbb{8}$ a TPG i.V.m. $\$ 8$ TPG normieren die allgemeinen Anforderungen im Hinblick auf die Minderjährigenspende.

\section{Das spendende Kind}

Allgemeine und für alle Fallgruppen geltende Voraussetzungen für eine Knochenmarkspende Minderjähriger sind zunächst Anforderungen an das spendende Kind. Dieses muss „nach ärztlicher Beurteilung als Spender geeignet“ sein und „voraussichtlich nicht über das Operationsrisiko hinaus gefährdet oder über die unmittelbaren Folgen der Entnahme hinaus gesundheitlich schwer beeinträchtigt" werden ( $\mathbb{8} 8$ Abs. 1 S. 1 lit. c TPG - Herv. v. Verf.). ${ }^{43}$ Damit gilt für Minderjährige im Hinblick auf das hinzunehmende eigene Opfer dieselbe Grenze wie für Volljährige. Das allgemeine Operationsrisiko ist insbesondere das der Narkotisierung. ${ }^{44}$ Schäden oder sogar Todeseintritt infolge der Narkose (vor allem aufgrund von Fehlbehandlungen sowie unerkannter Vorschäden des Kindes) sind heute sehr selten, aber nicht völlig auszuschließen. ${ }^{45}$ Neben dem allgemeinen Operationsrisiko darf keine schwere ge-

42 Vgl. $\$ 78$ BVerfGG.

43 Die Bestimmungen von $\mathbb{} 8$ TPG leuchten dort wieder auf, wo $\mathbb{} 8$ a TPG nichts Abweichendes regelt (vgl. $\$ 8$ Abs. 1 TPG).

44 Vgl. zum Begriff „Operationsrisiko“ bei der Lebendorganspende: D. Esser in: W. Höfling (Hrsg.), Kommentar zum Transplantationsgesetz, Berlin 2003, $\mathbb{8}$ Rn. 42.

45 J. Schimpf/ D. Craß/ V. Sollmann, Kompendium Kinderanästhesie, Berlin/Heidelberg 2012. „Komplikationen der Narkose - Narkosezwischenfälle - sind extrem selten, können aber nicht $100 \%$ ig ausgeschlossen werden.“ (http://www.stammzellspende.at/main.asp?content=spende-grundsaetze.asp). 
sundheitliche Beeinträchtigung zu erwarten sein, die über die unmittelbaren Folgen der Entnahme hinausreicht. Damit sind mittlere und leichte gesundheitliche Beeinträchtigungen zulässig.

Für die Knochenmarkspende wird das weitere Risiko meist nur mit muskelkaterähnlichen Wundschmerzen und Schmerzen an den Punktionsstellen, möglichen Blutergüssen, Müdigkeit ${ }^{46}$ sowie dem Infektionsrisiko ${ }^{47}$ angegeben. Das Knochenmark regeneriert sich in kurzer Zeit wieder. Die gängigen Angaben sind jedoch nicht zweifelsfrei, da Komplikationen nicht so freimütig publiziert werden wie ärztliche Erfolge. ${ }^{48}$ Auch gehört es heute zum common knowledge, dass Kinder nicht einfach nur kleine Erwachsene sind und daher die allgemeinen Risiken nicht einfach auf Kinder herunter gerechnet werden können. Interessant ist in diesem Zusammenhang, dass bei Erwachsenen unabhängig ihrer Körpergröße darauf geachtet wird, dass die Person mindestens $50 \mathrm{~kg}$ wiegt, ${ }^{49}$ also eine Vorgabe, die bei Kindern nicht gemacht wird. Völlig aus dem Blick geraten sind die psychischen Risiken, die sich für Kinder ganz anders darstellen als für Erwachsene. Es wird beschrieben, dass ein hohes psychisches Risiko besonders dann besteht, wenn die Spende nicht zum Erfolg führt. ${ }^{50}$ Aber auch schon der Krankenhausaufenthalt selbst und die damit verbundenen Ängste können die psychische Gesundheit antasten.

Bei klinischen Arzneimittelprüfungen an Minderjährigen erlaubt der Gesetzgeber wesentlich weniger Belastungen. Für eine Arzneimittelprüfung an gesunden Kindern bestimmt $\$ 40$ Abs. 4 Nr. 4 AMG, dass diese nur durchgeführt werden darf, „,wenn sie für die betroffene Person mit möglichst wenig Belastungen und anderen vorhersehbaren Risiken verbunden ist“. Diese Bestimmung ist in Umsetzung von Art. 4 lit g der RL 2001/20/EG ergangen, wonach klinische Prüfungen an Minderjährigen nur „mit möglichst wenig Schmerzen, Beschwerden, Angst und anderen vorhersehbaren Risiken verbunden“ sein dürfen. Bei der klinischen Prüfung an kranken Minderjährigen im Rahmen eines gruppennützigen Prüfungsziels gibt der Gesetzgeber mit $\ 41$ Abs. 2 Nr. 2 d AMG vor, dass „,die Forschung ... für die betroffene Person nur mit einem minimalen Risiko und einer minimalen Belastung verbunden sein [darf]“.

46 http://www.stammzellspende.at/main.asp?content=spende-grundsaetze.asp.

47 http://www.knochenmarkspende-deutschland.de/informationen_fuer_stammzellspender/risiken_einer_spende.php.

48 Vgl. auch Schmidt-Recla, Kontraindikation, (Fn. 7), S. 567: „Vielmehr ist anzunehmen, dass in schlecht verlaufenden Fällen keiner der Beteiligten - weder Ärzte noch Eltern - daran interessiert ist, Fehler rechtlich klären zu lassen.“

49 Zentrales Knochenmarkspenderregister Deutschland; http://www.zkrd.de/de/informationen_fuer_knochenmarkspender/ausschlusskriterien.php (Andere verlangen sogar ein Mindestgewicht von 55 kg: http://www.stammzellspende.at/main.asp?content=spende-grundsaetze.asp.

50 Roell, Geschwister (Fn. 18), S. 22 ff. 
Erklärend wird hinzugefügt:

„die Forschung weist nur ein minimales Risiko auf, wenn nach Art und Umfang der Intervention zu erwarten ist, dass sie allenfalls zu einer sehr geringfügigen und vorübergehenden Beeinträchtigung der Gesundheit der betroffenen Person führen wird; sie weist eine minimale Belastung auf, wenn zu erwarten ist, dass die Unannehmlichkeiten für die betroffene Person allenfalls vorübergehend auftreten und sehr geringfügig sein werden."

Die Zentrale Ethikkommission bei der Bundesärztekammer ist der Auffassung, dass von einem „minimalen Risiko“ nur dann gesprochen werden kann,

„wenn z. B. Körperflüssigkeit oder Gewebe in geringen Mengen im Rahmen von ohnehin notwendigen diagnostischen Maßnahmen oder Operationen gewonnen wird und deshalb kein zusätzliches Risiko für den Patienten beinhaltet. $"$ 51

Auch im Schrifttum finden sich als explizit zulässige Maßnahmen nur solche, die weit unter der Schwere einer nicht indizierten Operation und Knochenmarkentnahme liegen. ${ }^{52}$ Das „minimale Risiko“ bzw. die „minimale Belastung“ darf im Rahmen von $\mathbb{4} 41$ Abs. 2 Nr. 2 AMG (gruppennützige Arzneimittelprüfung an kranken Minderjährigen) nicht durch eine Verhältnismäßigkeitsprüfung dergestalt aufgeweicht werden, dass ein höherer Nutzen als rechtfertigend für eine höhere Belastung angesehen wird. ${ }^{53}$

Im Vergleich zu einer Arzneimittelprüfung an Kindern wird den Kindern im Rahmen einer Knochenmarkspende nach $\$ \mathbb{S 8 , 8}$ a TPG damit ein deutlich höheres Opfer abverlangt.

\section{Die Spendesituation und Durchführung}

Weiterhin gelten als Anforderungen an die Spendesituation, dass die „Spende nur für Verwandte ersten Grades ${ }^{54}$ oder Geschwister" erbracht werden darf $(\mathbb{8} 8$ a Nr. 1 TPG), ${ }^{55}$ dass „die Übertragung des Knochenmarks auf den vorgesehenen Empfänger

51 Zentrale Ethikkommission bei der Bundesärztekammer, Deutsches Ärzteblatt (94, Heft 15) 1997, S. 811.

52 S. Listl, in: A. Spickhoff (Hrsg.), Medizinrecht, München 2011 \41 AMG Rn. 4, zählt etwa auf: „EKG, EEG und Lungenfunktionsdiagnostik sowie vergleichbar riskante und belastende Maßnahmen“ neben Laborkontrollen und die hierfür erforderlichen Maßnahmen wie Urinproben bis hin zu „Blutentnahmen aus ohnehin vorhandenen Verweilkanülen und - je nach Alter und Konstitution des Minderjährigen - Venenblutentnahmen“.

53 Listl (Fn. 52) \$ 41 AMG Rn. 4.

54 Verwandte ersten Grades sind Eltern und Kinder, $\$ 1589$ BGB.

55 „Inwieweit bei dieser Beschränkung des Empfängerkreises einerseits das natürliche Füreinandereinstehen der engsten Verwandten und andererseits der verfassungsrechtlich vor staatlicher Einflussnahme geschützte Freiraum der Familie eine Rolle gespielt hat, ist den Materialien nicht zu entnehmen." (Schmidt-Recla, Kontraindikation (Fn. 7), S. 569). 
... nach ärztlicher Beurteilung geeignet [ist], bei ihm eine lebensbedrohende Krankheit zu heilen" ( $\$ 8$ a Nr. 2 TPG) sowie dass kein anderer volljähriger und einwilligungsfähiger Spender zur Verfügung steht ( $\$ 8$ a Nr. 3 TPG). Weiter gelten als $A n$ forderungen an die Art und Weise der Durchführung, dass „der Eingriff durch einen Arzt vorgenommen wird“ ( $\$ 8$ Abs. 1 Nr. 4 TPG), eine Aufklärung der gesetzlichen Vertreter durch einen Arzt stattgefunden hat $(\$ 8 \text { Abs. } 2 \text { TPG })^{56}$ sowie die Einwilligung des gesetzlichen Vertreters ( $\$ 8$ a Nr. 4 TPG). Das positive Votum der Lebendspendekommission nach $\mathbb{\$} 8$ Abs. 3 TPG ist dem Wortlaut nach nicht erforderlich, da die Knochenmarkspende keine Organspende ist.

\section{Das Kindeswohl}

Schließlich gilt nach $\mathbb{\$} 8$ a Nr. 4 TPG i.V.m. $\mathbb{1 6 2 7}$ BGB, dass die Ausübung der elterlichen Sorge, hier also die Einwilligung, zum Wohl des Kindes zu erfolgen hat. Der Kindeswohlbegriff passt allerdings seiner Natur nach nicht in eine fremdnützige Spende. So heißt es bei Kern, dass die Eltern grundsätzlich bei ihrer Einwilligung in medizinische Eingriffe „an das Kindeswohl gebunden“ sind, das „mit der medizinischen Indikation gleichzusetzen ist", woraus folge, dass nur in indizierte Eingriffe eingewilligt werden dürfe. ${ }^{57}$ Die Legitimation für eine juristische Erlaubnis der Fremdbestimmung bei Eingriffen in höchstpersönliche Rechte erhalte ihre Berechtigung aus der „medizinischen Notwendigkeit“, also dadurch, dass sie „dem Wohl des Betroffenen " dient. ${ }^{58}$

Eine spezialgesetzliche Ausnahmen hiervon gibt es bei der bereits erwähnten gruppennützigen klinischen Prüfung von Arzneimitteln an kranken Minderjährigen nach

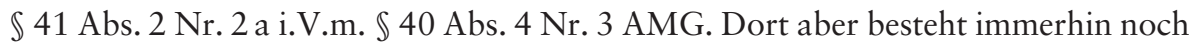
ein Bezug zum eigenen Leiden des Kindes und es ist nicht auszuschließen, dass das

$56 \$ 8$ Abs. 2 TPG: „Der Spender ist durch einen Arzt in verständlicher Form aufzuklären über 1. den Zweck und die Art des Eingriffs,

2. die Untersuchungen sowie das Recht, über die Ergebnisse der Untersuchungen unterrichtet zu werden,

3. die Maßnahmen, die dem Schutz des Spenders dienen, sowie den Umfang und mögliche, auch mittelbare Folgen und Spätfolgen der beabsichtigten Organ- oder Gewebeentnahme für seine Gesundheit, 4. die ärztliche Schweigepflicht,

5. die zu erwartende Erfolgsaussicht der Organ- oder Gewebeübertragung und sonstige Umstände, denen er erkennbar eine Bedeutung für die Spende beimisst, sowie über

6. die Erhebung und Verwendung personenbezogener Daten.

Der Spender ist darüber zu informieren, dass seine Einwilligung Voraussetzung für die Organ- oder Gewebeentnahme ist. Die Aufklärung hat in Anwesenheit eines weiteren Arztes, für den $\$ 5$ Abs. 2 Satz 1 und 2 entsprechend gilt, und, soweit erforderlich, anderer sachverständiger Personen zu erfolgen. Der Inhalt der Aufklärung und die Einwilligungserklärung des Spenders sind in einer Niederschrift aufzuzeichnen, die von den aufklärenden Personen, dem weiteren Arzt und dem Spender zu unterschreiben ist. Die Niederschrift muss auch eine Angabe über die versicherungsrechtliche Absicherung der gesundheitlichen Risiken nach Satz 1 enthalten. Die Einwilligung kann schriftlich oder mündlich widerrufen werden. Satz 3 gilt nicht im Fall der beabsichtigten Entnahme von Knochenmark."

57 Kern, Fremdbestimmung (Fn. 28), S. 756; ders., arzneimittelrechtliche Forschung (Fn. 3), S. 137. 
Kind als „Gruppenzugehöriger“ von der Arzneimittelprüfung profitiert. ${ }^{59}$ Es genügt nicht, dass die Prüfung im Hinblick auf irgendwelche Kinderkrankheiten stattfindet ${ }^{60}$ sondern der Minderjährige muss an der gleichen Krankheit leiden, wie die Gruppe der Patienten, für die der Test mit einem direkten Nutzen verbunden ist. ${ }^{61}$

In $\mathbb{S} 8$ a TPG ergibt sich nun die besondere Situation, dass am Kindeswohlkriterium explizit durch Verweis auf $\mathbb{} 1627$ BGB festgehalten und gleichwohl ein fremdnütziger Eingriff gestattet wird. Es stellt sich daher die ebenso schwierige wie weitreichende Frage, ob es das Ziel der Spende sein muss, dem Kindeswohl zu „dienen“ oder ob das Kindeswohl nur „nicht beeinträchtigt“ werden darf.

\section{a) Definition des Kindeswohls nach § 8 a TPG}

\section{aa) Problematik der Definition des Kindeswohls}

Der Kindeswohlbegriff ist unbestimmt und verlangt nach einer Auslegung. ${ }^{62}$ Verfassungsrechtlich wird das Kindeswohl nebulös beschrieben als zu konkretisieren durch die „Wertentscheidungen der Grundrechte“ sowie „das in den Grundrechten zum Ausdruck gelangende Menschenbild“. ${ }^{63}$ Das Grundgesetz zitiert das Kindeswohl nicht dem Begriff nach. Es ist allerdings allgemein anerkannt, dass sich sowohl das Elternrecht als auch das staatliche Wächteramt nach Art. 6 Abs. 2 GG am Kindeswohl zu orientieren haben. ${ }^{64}$ Für eine positive Umschreibung werden Art. 1 Abs. 1 GG (Menschenwürde) und Art. 2 Abs. 1 GG (allgemeine Handlungsfreiheit)

59 A. Spickhoff, Forschung an nicht-einwilligungsfähigen Notfallpatienten, MedR 2006, S. 707 (710): „,gruppenindividuelles‘ Wohl“; A.A. Kern, arzneimittelrechtliche Forschung, (Fn. 3), S. 144 (Gruppennutzen sei in Wahrheit ein reiner Fremdnutzen) mit weiteren Nachweisen.

60 Damit ist eine Arzneimittelprüfung nur unter engeren Voraussetzungen möglich als dies die Arzneimittelrichtlinie 2001/20/EG in Art. 4 lit. e vorsieht („Außerdem müssen sich derartige Forschungen unmittelbar auf einen klinischen Zustand beziehen, unter dem der betroffene Minderjährige leidet, oder ihrem Wesen nach nur an Minderjährigen durchgeführt werden können" -Herv v. Verf.). Näher hierzu Ch. Katzenmeier, in: A. Laufs/Ch. Katzenmeier/V. Lipp (Hrsg.), Arztrecht, München 2009, S. 490.

61 Eltern (als gesetzliche Vertreter) dürfen darüber hinaus im Hinblick auf ihre gesunden Kinder nur dann zu einer Teilnahme an der Prüfung von Arzneimitteln zustimmen, wenn das Arzneimittel dazu dienlich sein kann, bei ihrem Kind Krankheiten zu erkennen oder ihr Kind vor Krankheiten zu schützen $(\mathbb{S} 40$ Abs. 4 Nr. 1 AMG), vgl. auch Katzenmeier, (Fn. 60), S. 490; T. M. Spranger, Fremdnützige Forschung an Einwilligungsunfähigen, Bioethik und klinische Arzneimittelprüfung, MedR 2001, S. 238 (245) mit zahlreichen weiteren Nachweisen in Fn. 98; Spickhoff, Forschung an nicht-einwilligungsfähigen Notfallpatienten (Fn. 59), S. 707 (709): „angezeigt“ meint „medizinisch indiziert“.

62 Vgl. K. Bauer, in: M. Herberger/M. Martinek/H. Rüßmann/ S. Weth (Gesamthrsg.), juris PraxisKommentar-BGB, 5. Aufl., Saarbrücken 2011, $\$ 1666$ BGB Rn. 25; Brüser, Grundrechte im Kindesalter (Fn. 20), S. 90; Zitelmann, Kindeswohl und Kindeswille (Fn. 21), S. 118 ff.

63 G. Robbers, in: H. v. Mangoldt/F. Klein/Ch. Starck (Hrsg.), Grundgesetz-Kommentar, Bd. I, 6. Aufl., München 2010, Art. 6 Rn. 145 f.

64 A. Mehlitz, Die rechtliche Zulässigkeit placebokontrollierter klinischer Prüfungen nach der 12 . Novelle des Arzneimittelgesetzes - zugleich ein Beitrag zum Verständnis der $\$ \mathbb{S} 40,41$ AMG, 2008, http:// www.diss.fu-berlin.de/diss/receive/FUDISS_thesis_000000003653, S. 183. 
sowie Art. 2 Abs. 1 i.V.m. Art. 1 Abs. 1 GG (allgemeines Persönlichkeitsrecht) genannt. ${ }^{65}$ Die Aufnahme des Kindeswohls in das BGB

„folgt der grundgesetzlichen Vorgabe, dass Kinder als Rechtssubjekte und Träger von Grundrechten anerkannt und nicht als Objekte in einem besonderen Gewaltverhältnis' den Eingriffen ihrer Personensorgeberechtigten ausgesetzt sind." 66

Wenn $\mathbb{8} 8$ a TPG auf $\mathbb{1 6 2 7}$ BGB $^{67}$ verweist, dann liest sich die Norm zunächst als Pflicht der Eltern zum gegenseitigen Einvernehmen. ${ }^{68}$ Den Gesetzesmaterialien nach ist der Gesetzesverweis aber vor allem als Ausdruck des Maßstabes „Kindeswohl“ gemeint. ${ }^{69}$ Das „Wohl“ des Kindes wird im BGB an zahlreichen Stellen benannt, wenn es um Entscheidungen geht, die (auch) das Kind betreffen. ${ }^{70}$ Hervorzuheben ist $\$ 1697$ a BGB ${ }^{71}$ der das „Kindeswohlprinzip“ normiert. In $\mathbb{1 6 6 6}$ (und $\$ 1666$ a BGB) geht es um das „negative Kindeswohl“, die Kindeswohlgefährdung.

Die Knochenmarkspende erfüllt - strafrechtlich betrachtet - den Tatbestand einer

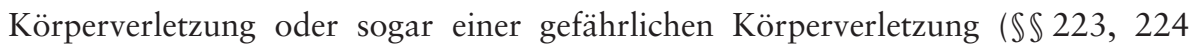
StGB). ${ }^{72}$ Legt man bezogen auf die Spendefälle das Kindeswohl so aus, dass dies bedeute, dass eine medizinische Indikation für das Spenderkind gegeben sein muss, so könnte das Kindeswohlkriterium in den Spendefällen nie erfüllt sein. ${ }^{73} \$ 8$ a TPG wäre dann eine in sich widersprüchliche Norm.

65 Vgl. B. Jeand'Heur, Verfassungsrechtliche Schutzgebote zum Wohl des Kindes und staatliche Interventionspflichten aus der Garantienorm des Art. 6 Abs. 2 Satz 2 GG, Berlin 1993, S. 17; P. Hölbling, Wie viel Staat vertragen die Eltern?, Berlin 2010, S. $143 \mathrm{f}$.

66 Schmidt-Recla, Kontraindikation (Fn. 7), S. 571.

$67 \int 1627$ BGB (Ausübung der elterlichen Sorge): „Die Eltern haben die elterliche Sorge in eigener Verantwortung und in gegenseitigem Einvernehmen zum Wohl des Kindes auszuüben. Bei Meinungsverschiedenheiten müssen sie versuchen, sich zu einigen."

68 Vgl. zur Frage, inwieweit bei einem Heileingriff zu Gunsten des Kindes beide Eltern zustimmen müssen: OLGR München 2009, 848 (red. Leitsatz und Gründe), abgedruckt in: FamRZ 2009, S. 2099 (red. Leitsatz und Gründe).

69 BT-Drs. 16/3146, S. 29.

70 Beispiele: $\ 1361$ b; 1568 a; 1568 b BGB (Kindeswohl als Grund für die Überlassung der Ehewohnung/ Haushaltsgegenstände bei Getrenntlebenden/Geschiedenen); $\mathbb{1 5 9 8}$ a Abs. 3 BGB (Klärung der Vaterschaft).

$71 \int 1697$ a BGB (Kindeswohlprinzip): „Soweit nichts anderes bestimmt ist, trifft das Gericht in Verfahren über die in diesem Titel geregelten Angelegenheiten diejenige Entscheidung, die unter Berücksichtigung der tatsächlichen Gegebenheiten und Möglichkeiten sowie der berechtigten Interessen der Beteiligten dem Wohl des Kindes am besten entspricht.“

72 Vgl. allgemein zur Einordnung selbst medizinisch indizierter ärztlicher Eingriffe als Körperverletzung durch die Rechtsprechung M. Nebendahl, Selbstbestimmungsrecht und rechtfertigende Einwilligung des Minderjährigen bei medizinischen Eingriffen, MedR 2009, S. 197 sowie oben Fn. 5.

73 Vgl. auch Schmidt-Recla, Kontraindikation (Fn. 7), S. 572. 
bb) Mögliche Kindeswohlinterpretationen im Rahmen von § 8 a TPG (i.V.m. § 1627 BGB)

Daher sollen im Folgenden zwei mögliche „sinnhafte“ Kindeswohlinterpretationen vorgestellt werden, die dann unter C I 3 b) auf ihre Verfassungsmäßigkeit hin analysiert werden.

Eine erste denkbare Interpretation, die $\mathbb{\int} 8$ a TPG mit einem sinnvollen Normgehalt füllt, bestünde darin, die Norm so auszulegen, dass das „Kindeswohlkriterium “ lediglich bedeutet, dass die Knochenmarkentnahme das Kind nicht über das Operationsrisiko hinaus gefährden darf, etwa aufgrund seines Alters, seiner Konstitution oder aufgrund von Vorerkrankungen. So gelesen würde das Kindeswohl die Anforderung aus $\mathbb{\int} 8$ Abs. 1 S.1 lit c TPG (auf die $\mathbb{S} 8$ a TPG verweist) noch einmal schärfen, denn dort wird nur bestimmt, dass neben dem allgemeinen Operationsrisiko die Spende den Spender nicht „gesundheitlich schwer beeinträchtigen“ darf. Mittlere Beeinträchtigungen wären für Volljährige zulässig, könnten nun aber über das Kindeswohl unzulässig sein. Dann wäre das „Kindeswohl“ allerdings kein positiver Begriff mehr, sondern würde lediglich „Abwehr großen und mittleren Schadens“ bedeuten. Es wäre danach nicht einmal notwendig, den physischen und psychischen Schaden, der durch die Spende entsteht, mit dem Nutzen der Spende aufzuwiegen.

Eine zweite Interpretation, die $\mathbb{\int} 8$ a TPG zu einer sinnvollen Norm erhebt, wäre daher, dass die Spende dem Spenderkind aufgrund der gesamten Familiensituation zum „positiven“ Wohl dient. Dahinter steht die Idee, dass das Kindeswohl nicht isoliert betrachtet werden kann, sondern das Familienwohl auch immer in Wechselwirkung zum Spenderkind steht. Gemeint wäre dann der Nutzen für die Psyche des Spenderkindes, welcher so schwer wiegt, dass er den physischen Eingriff aufwiegen könnte. Dieses Wohl müsste dann aber für jede Spendesituation gesondert festgestellt werden. Nach der Gesetzessystematik von $\mathbb{\int} 8$ a TPG i.V.m. $\mathbb{S} 1627$ BGB sind es die Eltern (gesetzlichen Vertreter), die dieses Wohl festzustellen haben.

b) Verfassungsmäßigkeit des Kindeswohlkriteriums

\section{aa) Verfassungsmäßigkeit der möglichen Kindeswohlinterpretationen}

Die erste vorgestellte Interpretation des Kindeswohls, die darauf abzielte, dass das Kindeswohl nur bedeute, dass lediglich ein großer oder mittlerer Schaden abgewendet werden soll, wäre problematisch, weil $\mathbb{\int} 8$ a TPG eine „staatlich legitimierte Eingriffsbefugnis“ des Arztes nach Einwilligung der Eltern darstellt und auf diese Weise der Schutz vor Körperverletzungen durch Dritte aufgeweicht wird. Solange die Eltern stellvertretend die Grundrechte für das Kind ausüben und stellvertretend die Spendeentscheidung fällen, ist eine Auslegung, nach der dem Kind ein - wenn auch ge- 
ringer - Schaden ohne aufwiegenden Nutzen zugefügt wird, nicht mit Art. 6 Abs. 2 GG vereinbar. ${ }^{74}$

Die zweite vorgestellte Kindeswohlinterpretation beachtet über das körperliche Wohl hinaus das psychische Wohl. Die Verfassung steht selbstverständlich nicht der Wahrung des psychischen Wohls des Kindes entgegen, sondern fordert diese nach Art. 6 Abs. 2 GG sogar ein. ${ }^{75}$ Es obliegt den Eltern (nach Art. 6 Abs. 2 GG) und in gewissem Umfang auch dem Staat (nach Art. 2 Abs. 2 und 6 Abs. 2 GG) eine Verpflichtung, das Kind auch vor körperlichem Schaden zu bewahren.

Geht man davon aus, dass das Spenderkind durch eine Genesung des Geschwisterkindes und die dadurch bewirkte Normalisierung der Familiensituation selbst psychisch profitiert, streiten im Spendefall die physische und die psychische Gesundheit des Spenderkindes gegeneinander, da der körperliche Eingriff rein physisch betrachtet nicht dem Wohl des Spenderkindes dient. Hier ist - wie oft im Verfassungsrecht - eine Abwägung im Einzelfall notwendig. Dies bedeutet, es kann nicht abstrakt vorweg festgestellt werden, ob die Pflicht zum Erhalt des körperlichen oder des seelischen Wohls überwiegt. Maßgebend sind vielmehr die konkreten Umstände des Einzelfalles, etwa die Schwere des körperlichen Eingriffs; die möglichen seelischen Folgen bei Fehlschlagen der Behandlung des kranken Geschwisterkindes; die zu erwartenden positiven psychischen Auswirkungen bei Heilung des kranken Geschwisterkindes etc.

Hier liegt das Problem in der Tatsachenfeststellung, die antizipieren muss, wie es dem Spenderkind ohne und mit einer Spende gehen würde. An dieser Stelle ist ein Defizit an empirischen Studien zu verzeichnen. Aus den wenigen Studien, die vorliegen, ergibt sich im Hinblick auf das Wohl des Spenderkindes ein auffallend verhaltenes Bild: Ergeben sich für das Empfängerkind Komplikationen oder stirbt es, wirkt sich dies äußerst negativ auf das Befinden des Spenderkindes aus. ${ }^{76}$ Es wird von ausgeprägten Schuldgefühlen berichtet, von Verzweiflung und Bedrängnis. Selbst bei positivem

74 Anders wohl T. Exner, Sozialadäquanz im Strafrecht, Berlin 2011, S. 42: „Mit dem Kindeswohl vereinbar kann vielmehr auch die ärztlich für den Minderjährigen nicht indizierte, jedoch in Erfüllung einer sittlichen oder sozialen Pflicht erfolgende Preisgabe kindlicher Rechtsgüter sein, wie etwa in Form einer Bluttransfusion für einen Verunglückten."

75 Das Kindeswohl ist der Erziehungsmaßstab für Art. 6 Abs. 2 GG durch die Eltern. „Pflichtbegründender Schutzzweck der Norm sind die spezifischen Schutz-und Fürsorgebedürfnisse der Kinder“ $(M$. Kotzur in: K. Stern/F. Becker, Grundrechte-Kommentar, 1. Aufl., Köln 2010, Art. 6 Rn. 86). Der Begriff des „Kindeswohls“ und der „Fürsorgebedürfnisse“ umfasst dabei nicht nur die Sorge für die physische Existenz, sondern auch für die gesunde psychische Entwicklung des Kindes. Ebenso ist das psychische Wohl des Kindes über Art. 2 Abs. 2 GG geschützt; Hölbling, Wie viel Staat vertragen die Eltern? (Fn. 65), S. 145.

76 Roell, Geschwister (Fn. 18), S. 22 ff. (mit Auswertung vorhandener Studien). Vgl. hierzu auch die Erkenntnisse, die für Lebendnierenspenden unter Volljährigen vorliegen, wonach bei Komplikationen bzw. Versterben des Empfängers Schuldgefühle bis hin zu Suizidgedanken beschrieben werden, vgl. A. Stoffers, Der Entscheidungsprozess im Kontext einer Lebendnierentransplantation aus Sicht von Spenderinnen und Spendern - eine qualitative Studie, Würzburg 2009, S. 14. 
Ausgang für das Empfängerkind zeigen Studien, dass das Spenderkind den Eingriff als „räuberischen Akt“ erlebt „der die Körperintegrität bedrohte“. ${ }^{77}$ Bei Grundschulkindern wurden „Ohnmachts- und Hilflosigkeitsgefühle“78 beobachtet sowie „Todesängste“ und „Vergiftungsphantasien“ und psychische Reaktionen, die auch ein Jahr nach der Spende noch bestanden (Trennungsangst, Verhaltensstörungen). ${ }^{79}$

Andererseits sind Spender auch „stolz“ auf ihre Hilfe und es kann auch eine Steigerung des Selbstwertgefühls verzeichnet werden. ${ }^{80}$ HLA-kompatible Geschwisterkinder, die nicht spenden durften, weil ein anderes Geschwisterkind etwa aufgrund seines Alters besser geeignet war, fühlten sich sogar gekränkt. ${ }^{81}$ Insgesamt ist es sehr schwer, zu beurteilen, welche Reaktionen der Spender tatsächlich auf der Spende beruhen und welche Reaktionen eine Folge der gesamten belasteten Familiensituation sind. ${ }^{82}$ Das Familiengefüge gerät nämlich nachhaltig aus den Fugen. Als besonders belastend wird die lange Abwesenheit der Mutter im Krankenhaus beim Empfängerkind beschrieben, durch die sich die anderen Kinder, ob Spender oder Nichtspender, vernachlässigt fühlen. Der Tod des kranken Kindes führt bei der Familie zu „Schockgefühlen“, Verzweiflung, Enttäuschung, Verleugnung und Wut, wobei die Spenderkinder ,sich trotzdem häufiger Selbstvorwürfe machten und den Tod mit ihrer Spende in Zusammenhang brachten “. ${ }^{83}$

Sollte nach einer Abwägung zwischen dem physischen Eingriff und dem antizipierten psychischen Wohl des Spenderkindes eine positive Bilanz gezogen werden können, dient der Eingriff also letztlich dem Kindeswohl auch des Spenderkindes, so wäre er verfassungsrechtlich unbedenklich.

Weiter steht in Frage, ob es auch ausreicht, dass lediglich kein Eingriff in das Kindeswobl zu erkennen ist, ob also auch eine „Null-Linie“ mit der Verfassung im Einklang stünde. Eine Legitimation für eine stellvertretende Elternentscheidung in einen körperlichen Eingriff kann sich aus dem Elternrecht nach Art. 6 Abs. 2 GG grundsätzlich nur ergeben, wenn es sich dabei um eine Entscheidung handelt, die im $\mathrm{Zu}$ sammenhang mit der Pflege und Erziehung des Kindes steht, was für den Fall eines medizinischen Eingriffs ohne Nutzen bereits fraglich wäre. Darüber hinaus kommt eine staatliche Schutzpflicht aus Art. 2 Abs. 2 GG in Betracht, wenn die gesetzliche Zulässigkeit einer fremdbestimmten Spende das unterschreitet, was als angemessener Schutz eines Kindes vom Staat verlangt werden kann. Unzureichend wäre der Schutz,

77 Roell, Geschwister (Fn. 18), S. 23.

78 Roell, Geschwister (Fn. 18), S. 23.

79 Roell, Geschwister (Fn. 18), S. 24 f. sowie S. 105 ff. Die Verhaltensstörungen wurden dabei nicht nur den jüngeren Spenderkindern zugeordnet (Studie ohne Altersangabe).

80 Roell, Geschwister (Fn. 18), S. 27, 107.

81 Roell, Geschwister (Fn. 18), S. 313.

82 Roell, Geschwister (Fn. 18), S. $31 \mathrm{ff.}$

83 Roell, Geschwister (Fn. 18), S. 322 f. (Zitat auf S. 323); vgl. auch S. 112. 
wenn der Staat explizit und gewollt einen unverhältnismäßigen Eingriff durch Dritte in die körperliche Unversehrtheit zulässt. ${ }^{84}$

Die Geeignetheit und Erforderlichkeit der Spende werden bereits durch die allgemeinen Spendevoraussetzungen abgesichert (Spender muss geeignet sein und es darf kein anderer volljähriger einwilligungsfähiger Spender zu Verfügung stehen). ${ }^{85} \mathrm{Al}$ lein in Frage steht also die Angemessenheit der Knochenmarkspende, bei der sich Nutzen und Schaden für das Spenderkind die Waage halten („Null-Linie“). Für die Angemessenheit spricht der lebensentscheidende Nutzen für das Empfängerkind bei „ausgeglichener Bilanz" für das Spenderkind. Gegen die Angemessenheit muss die „Verzweckung“ des Spenderkindes angeführt werden, solange die Grundrechtsausübung vollständig von den Eltern fremdbestimmt wird (Fallgruppe „Baby“). Kein Erwachsener darf zu einer Knochenmarkspende ( $\$ 8$ Abs. 1 Nr. 1 b TPG) oder auch nur Blutspende (vgl. $\$ 6$ Abs. 1 Transfusionsgesetz) gezwungen werden, selbst wenn diese für einen nahen Angehörigen lebensrettend wäre. Würden wir also die KostenNutzen-Abwägung hier als maßgeblich betrachten, müssten wir konsequenterweise auch andernorts über eine Spendepflicht nachdenken (etwa für erwachsene Geschwisterkinder oder auch darüber hinaus). Eine solche Verzweckung, bei der das Spenderkind ohne oder gegen seinen Willen als Objekt, als „Ersatzteillieferant“ gebraucht wird, verstößt überdies gegen Art. 1 Abs. 1 GG. ${ }^{86}$ Die Freiwilligkeit der Spende ist also ein zentrales Element, die grundsätzlich nur dann zurücktreten kann, wenn die Spende dem Wohl des Spenderkindes dient.

\section{bb) Zur Entscheidungsbefugnis der Eltern über das Vorliegen des „Kindeswohls“}

Wer befindet nun darüber, ob der Eingriff dem Kindeswohl dient oder für das Kindeswohl schädlich ist? Zunächst obliegt die Sorge für das Kind den Eltern (Art. 6 Abs. 2 GG). Das Elternrecht ist umso stärker, je weniger handlungs- und entschei-

84 Zum Untermaßverbot vgl. C.- W. Canaris, Grundrechte und Privatrecht, AcP 184 (1984), S. 201 (228); BVerfGE 88, 203, (LS 6).

85 An dieser Stelle könnte aus medizinischer Sicht analysiert werden, ob die periphere Blutstammzellspende ein weniger starker oder sogar belastenderer Eingriff für das Spenderkind wäre. Bislang ist nicht gänzlich geklärt, welche Nebenwirkungen für Kinder zu befürchten sind (vgl. Nachweise (Fn. 2)).

86 Vgl. für die medizinische Arzneimittelforschung an Kindern und Einwilligungsunfähigen: Mehlitz, Die rechtliche Zulässigkeit placebokontrollierter klinischer Prüfungen (Fn. 64), S. 155 ff., 177. Spickhoff, Forschung an nicht-einwilligungsfähigen Notfallpatienten (Fn. 59), S. 710; Spranger, Fremdnützige Forschung (Fn. 61), S. 243; M. Wunder, Unrecht durch Ungleichbehandlung oder Gleichbehandlung im Unrecht? JZ 2001, S. 344 (345). 
dungsfähig das Kind ist. ${ }^{87}$ Die Steigerung der Grundrechtsausübungsfähigkeit des Kindes bedingt das Abnehmen des Elternrechts. Das Elternrecht besteht überdies zugunsten des Kindeswohls und kann daher nicht in Konkurrenz zum Kindeswohl treten. ${ }^{88}$ Art. 6 Abs. 2 S. 1 GG ist für die Eltern zugleich Grundrecht und Grundpflicht. ${ }^{89}$ Das „Wächteramt“ des Staates tritt hinzu, wenn Anlass zur Annahme besteht, dass die Eltern die Fürsorgepflicht nicht zum Wohle des Kindes ausüben. ${ }^{90}$ Aus diesem Grunde sieht $\$ 8$ a TPG auch für die Spende zugunsten eines Elternteils eine Entscheidung des Familiengerichts über eine mögliche Entziehung der Vertretungsmacht der Eltern vor.

Eine ähnliche Besorgnis wie bei einer Spende für das Elternteil selbst besteht aber auch bei einer Spende für ein Geschwisterkind. Denn die Eltern fühlen sich zugleich dem Wohl des kranken Kindes wie des potentiellen Spenderkindes verpflichtet. Sie stehen in einem Interessenskonflikt, und es besteht Anlass zur Sorge, dass sie aus diesem Grunde das potentielle Spenderkind nicht mehr so vertreten können, dass sie sein Wohl unabhängig vom Wohl des kranken Kindes beurteilen können. Es wird sogar von einer „präjudizierten Eingriffsermächtigung in die Zwangsbehandlung des Spenderkindes, ohne dass eine Interessenabwägung " stattfindet, gesprochen. ${ }^{91}$ Der „Wächtergedanke“ des Staates aus Art. 6 Abs. 2 S. 2 GG muss hier zum Zuge kommen. Die Entscheidungsbefugnis über das Wohl des Spenderkindes allein den Eltern zu übertragen, weckt daher Zweifel.

\section{cc) Zum Kindeswohlkriterium in Abhängigkeit von der Einsichtsfähigkeit}

Mit der zunehmenden Einsichtsfähigkeit muss das Kind berechtigt werden, selbst mehr über seinen Körper zu entscheiden. Überdies tritt die Pflicht des Staates, das Kind zu schützen, zunehmend in den Hintergrund, wenn diese Pflicht mit der autonomen Entscheidung eines einsichtsfähigen Kindes kollidiert. Auch das Elternrecht aus Art. 6 Abs. 2 GG ist, wie gezeigt, an der zunehmenden Reife des Kindes auszurichten und darf nicht mehr zum vermeintlichen Wohle gegen jeden Widerstand durchgesetzt werden. Es scheint also verfassungsrechtlich möglich oder sogar gebo-

87 B. Pieroth, in H. D. Jarass/B. Pieroth (Hrsg.), Grundgesetz-Kommentar, 11. Aufl., München 2011, Art. 6 Rn. 38: „Zeitliche Abstufungen. Die im Elternrecht wurzelnden Rechtsbefugnisse beginnen mit der Geburt, nehmen mit fortschreitendem Alter des Kindes ab und erlöschen mit der Volljährigkeit des Kindes.“; Golbs, Das Vetorecht (Fn. 4), S. 87 ff.; Robbers (Fn. 63), Art. 6 Rn. 159; Gröschner (Fn. 38), Art. 6 Rn. 115; Brüser, Grundrechte im Kindesalter (Fn. 20), S. 65 f.; BVerfGE 72, 122 (137); vgl. auch $\int 1626$ Abs. 2 BGB: „Bei der Pflege und Erziehung berücksichtigen die Eltern die wachsende Fähigkeit und das wachsende Bedürfnis des Kindes zu selbständigem verantwortungsbewusstem Handeln. Sie besprechen mit dem Kind, soweit es nach dessen Entwicklungsstand angezeigt ist, Fragen der elterlichen Sorge und streben Einvernehmen an."

88 Coester-Waltien (Fn. 38), Art. 6 Rn. 81.; Jeand'Heur, Verfassungsrechtliche Schutzgebote (Fn. 65), S. 18.

89 Jeand'Heur, Verfassungsrechtliche Schutzgebote (Fn. 65), S. 20.

90 Kindeswohl als Schutzpflicht des Staates, s. Gröschner (Fn. 38), Art. 6 Rn. 100, 121.

91 Gerdts, $\mathbb{S} 8$ a TPG (Fn. 12), Abschnitt VI. 
ten, den Kindeswohlbegriff des $\mathbb{8}$ a TPG in Abhängigkeit von der Einsichtsfähigkeit unterschiedlich auszulegen, da die paternalistische Bestimmung des Wohls der Autonomie des Kindes entgegensteht. Das Kindeswohl als Baustein für die Subjektstellung und Interessenwahrnehmung des Kindes soll nur die fehlende freiwillige und mündige Entscheidung überbrücken, muss also graduell zurücktreten oder in seinem Gehalt anders verstanden werden, wenn die Entscheidung des Kindes auf Einsichtsfähigkeit beruht und damit von einer echten Freiwilligkeit gesprochen werden kann.

\section{Verfassungsmäßigkeit der gesetzlichen Fallgruppen des § 8 a TPG}

Im Folgenden sollen die oben erarbeiteten verfassungsrechtlichen Maßstäbe systematisch auf die einzelnen Fallgruppen angewendet werden.

\section{Fallgruppe: Spendewillige Eltern und Baby}

Geben die Eltern ihre Einwilligung, so darf nach $\$ 8$ a Nr. 4 TPG ein Baby als Knochenmarkspender für sein Geschwisterkind gebraucht werden, sofern die weiteren Tatbestandsvoraussetzungen (oben C I) erfüllt sind. Aus verfassungsrechtlicher Sicht muss hier, da das Kind noch gar keinen eigenen Willen in Bezug auf seine Spende bilden kann, besonders vorsichtig abgewogen werden, ob das „Kindeswohlkriterium “, das die mangelnde Freiwilligkeit und Einsichtsfähigkeit des Babys überbrücken soll, erfüllt ist. Im Rahmen der Abwägung, ob die Spende auch dem Wohl des Spenderbabys dient, muss insbesondere auch in Erwägung gezogen werden, inwieweit eine Knochenmarkentnahme für ein Baby medizinisch risikoreicher verläuft als bei älteren Kindern. Eine Knochenmarkspende dürfte überdies nur dann durchgeführt werden, wenn aufgrund der besonderen Fallumstände mit überwiegender Wahrscheinlichkeit von einem positiven (psychischen) Nutzen für das Spenderbaby ausgegangen werden kann.

Ob das Ablehnungsrecht nach $\mathbb{\$} 8$ a Nr. 4 TPG gilt, ist ungeklärt. ${ }^{92}$ Würde man $\mathbb{S} 8$ a Nr. 4 TPG so lesen, dass bereits dem unverständigen Baby ein Ablehnungsrecht zustünde, so schiede ein schreiendes oder verängstigt wirkendes Baby schon aus diesem Gesichtspunkt in den meisten Fällen als Spender aus. Die Reaktion des Babys ist sachgerecht in der Beurteilung des Kindeswohls zu berücksichtigen, nicht aber als eigenes (eingeschränktes) Vetorecht zu fassen.

Wie bereits erörtert, dürfen es aus verfassungsrechtlicher Sicht nicht allein die Eltern sein, die über das Kindeswohlkriterium entscheiden, da sie in einem Interessenskonflikt stehen (s. Vorschlag einer Kommission unter D II).

92 Kern, arzneimittelrechtliche Forschung (Fn. 3), S. 147. Schmidt-Recla, Kontraindikation (Fn. 7), S. 569 („Das Vetorecht betrifft Situationen, in denen das Kind zwar noch nicht positiv einwilligungsfähig, aber bereits so entwickelt ist, dass eine Entscheidung nicht mehr vollständig ohne es gefällt werden darf.“). 


\section{Fallgruppe: Spendewillige Eltern und Grundschulkind}

a) Aufklärung des Grundschulkindes

Im Falle der Spende durch ein (wie oben definiertes) Grundschulkind ist nach $\$ 8$ a Nr. 4 TPG zusätzliche Voraussetzung, dass das Kind durch einen Arzt aufzuklären ist, „soweit dies im Hinblick auf [...] Alter und [...] geistige Reife möglich ist. “ Wann dies der Fall ist, wird nicht näher konkretisiert. ${ }^{93}$

Diese altersgerechte Aufklärung zielt auf die Willensbildung des Kindes ab. Entscheidend wäre (die nicht normierte Voraussetzung), dass die Aufklärung von einem „objektiven“ Arzt vorgenommen wird und nicht von dem Arzt, der das Empfängerkind betreut (Rollenklarheit). Anderenfalls ist ein Interessenskonflikt des Arztes zu befürchten, so dass die Aufklärung auch zu einer „Überredung“ werden kann. Die Aufklärung, die an sich die Interessen des Spenderkindes schützen soll, könnte dann in ihr Gegenteil verkehrt werden und im schlimmsten Falle das Spenderkind unter Druck setzen (etwa wenn der Arzt das Kind eindringlich darauf hinweist, dass die Stammzellspende die letzte Chance für das Überleben seines Geschwisterkindes ist). Damit würde das Kind nicht nur in der Bildung seines freien Willens empfindlich beeinträchtigt, sondern es könnte auch psychische Schäden davon tragen, wenn ihm eine solche Verantwortlichkeit für das Wohl des Geschwisterkindes übertragen wird. Eine Ablehnung kann zu „massiven Schuldgefühlen“ führen, indem das Kind annimmt, sein Geschwister „ermordet“ zu haben. ${ }^{94}$ Die Aufklärung kann sogar dazu führen, dass das Kind keine Möglichkeit hat, „unverletzt“ aus der Situation herauszukommen (da es entweder die Verletzung durch die Spende hinnehmen muss oder die Verletzung durch die massiven Schuldgefühle). Die Aufklärung muss der Subjektqualität des Kindes gerecht werden. ${ }^{95}$

\section{b) Ablehnung der Spende durch das Grundschulkind}

Lehnt nun das Grundschulkind die Entnahme ab, sei es durch Worte oder Gestik, so ist dies nach $\mathbb{\int} 8$ a Nr. 4 S. 4 TPG „zu beachten“. Eine ähnliche Bestimmung findet sich bereits in $\mathbb{S} 40$ Abs. 4 Nr. 3 S. 3 AMG, also bei dem Einbezug Minderjähriger in

93 Vgl. auch Th. Roth, in: D. Prütting (Hrsg.), Fachanwaltskommentar Medizinrecht, 2. Aufl., Köln 2012, $\mathbb{~} 8$ a TPG Rn. 9: Mangels Maßstäben wird die Beurteilung „im Einzelfall schwer fallen, was letztlich darauf hinausläuft, den potentiellen Spender im Zweifel vollständig, wie einen Volljährigen aufzuklären."

94 Roell, Geschwister (Fn. 18) S. 24.

95 Vgl. auch Th. Moos, Theologisch-ethische Überlegungen zum fremdnützigen medizinischen Eingriff bei Kindern (Tagungsbeitrag Lübeck November 2011); A. K. Weilert, Grundlagen und Grenzen des Folterverbotes in verschiedenen Rechtskreisen, Heidelberg 2009, S. 135. Für weitere inhaltliche Anforderungen Roell, Geschwister (Fn. 18), S. 325. 
klinische Prüfungen. ${ }^{96}$ Unklar ist bereits, von wem die Ablehnung zur Kenntnis genommen werden muss. Für die analoge Vorschrift des $\mathbb{} 40$ Abs. 4 Nr. 3 S. 3 AMG wird wohl teils davon ausgegangen, dass sowohl der Arzt als auch der gesetzlicher Vertreter die kindliche Ablehnung zu beachten haben. ${ }^{97}$

Überträgt man eine solche Auslegung auf $\mathbb{S} 8$ a TPG, würde das Spenderkind am intensivsten vor einer fremdnützigen Zwangsbehandlung geschützt werden. Dogmatisch könnte man einwenden, dass der Kindeswille nur gegenüber den Eltern relevant sein kann, die allein einwilligungsberechtigt sind, und dass die ablehnende Haltung des Kindes lediglich ihre Vertretungsmacht einschränkt. ${ }^{98}$

Andererseits ist der Wortlaut offen und erlaubt auch die Auslegung, dass dem Kind eine Art „Vetorecht“ auch gegenüber dem Arzt zusteht, so dass er eine ihm gegenüber gezeigte ablehnende Reaktion nicht einfach übergehen darf. Nun würde dies allerdings für den Arzt eine gewisse Rechtsunsicherheit bedeuten, indem er im Spannungsverhältnis der Einwilligung der Eltern und seiner eigenen Wertung der Reaktion des Kindes stünde. Diese Spannung wäre insbesondere dann gravierend, wenn die Ablehnung nicht zwingend zum Ausschluss der Spende führte. Dem könnte etwa dadurch abgeholfen werden, dass man $\mathbb{S} 8$ a Nr. 4 TPG so versteht, dass der Arzt seine Zweifel lediglich den Eltern mitzuteilen hat, die dies in ihre Entscheidung einzubeziehen haben oder ihre bereits gegeben Einwilligung noch einmal prüfen müssen. Ebenso könnte man das Spannungsverhältnis entschärfen, wenn dem Arzt die Möglichkeit eingeräumt würde, für diesen Fall eine Kommission anzurufen (dazu näher unten).

Schon für $\ 40$ Abs. 4 Nr. 3 S. 3 AMG besteht Unklarheit darüber, wie das Wort „zu beachten" auszulegen ist. Nach S. Listl wird die Beachtung eines entgegenstehenden Willens so verstanden,

„dass eine eindeutige, ausdrücklich oder konkludent zum Ausdruck gebrachte Verweigerung die Prüfungsteilnahme verhindert, es sei denn, aus dem Entwicklungsstand, dem sonstigen Verhalten und der daraus erkennbaren Grundhaltung des Minderjährigen heraus ist ersichtlich, dass die Verweigerung nicht seinem wohlverstandenen, mutmaßlichen Willen entspricht, sondern vielmehr

96 S. auch den Verweis in $\$ 41$ Abs. 3 Nr. 2 S. 2 AMG auf $\$ 40$ Abs. 4 Nr. 3. S. 3 AMG für nichteinwilligungsfähige volljährige Personen. Auf internationaler Ebene schützt Art. 20 Abs. 2 lit v des Übereinkommens zum Schutz der Menschenrechte und der Menschenwürde im Hinblick auf die Anwendung von Biologie und Medizin: Übereinkommen über Menschenrechte und Biomedizin des Europarates vom 4. April 1997 davor, dass eine nicht einwilligungsfähige Person trotz einer Ablehnung zur Spende von regenerierbarem Gewebe herangezogen wird.

97 Kern, arzneimittelrechtliche Forschung (Fn. 3), S. 147 f.

98 In diese Richtung wohl Roth, (Fn. 93), $\$ 8$ a TPG Rn. 10. 
etwa auf kindliche Abwehrreaktionen gegen Unbekanntes zurückzuführen ist. " 99

Der „wohlverstandene mutmaßliche Wille “ bedeute dabei, dass entscheidend nicht der mutmaßliche Wille allein ist, sondern dieser übergangen werden kann, wenn „ein besonderer Nutzen für den Minderjährigen zu erwarten ist oder aus der Studienteilnahme gegenüber dem Nutzen nur minimale Risiken resultieren “. ${ }^{100}$ Auf Bedenken stößt allerdings bereits das Konzept eines mutmaßlichen Willens, der normalerweise nur dann zum Tragen kommt, wenn ein geäußerter Wille fehlt. ${ }^{101}$ Hier aber liegt in der Ablehnung bereits ein „geäußerter Wille“, dessen „rechtliches Gewicht“ allein in Frage steht. Listl operiert vor diesem Hintergrund mit dem „wohlverstandenen“ mutmaßlichen Willen, der letztlich gerade auf die Überwindung des tatsächlich geäußerten Willens abzielt und daher auch treffender als Fremdbestimmung (ggf. zum Wohle des Kindes) charakterisiert werden sollte. B.-R. Kern versteht $\mathbb{} 40$ Abs. 4 Nr. 3 S. 3 AMG im Sinne eines Vetorechts, jedenfalls solange nicht die Behandlung „im Interesse des Kindes dringend geboten“ ist. ${ }^{102}$ Diese Auslegung steht auch in Übereinstimmung mit den Gesetzesmaterialien. ${ }^{103}$

Für $\ 8$ a Nr. 4 S. 4 TPG ist in systematischer Auslegung festzuhalten, dass eine ablehnende Haltung nicht bedeutet, dass der Eingriff nicht vorgenommen werden darf (im Unterschied zur erforderlichen Einwilligung des Teenagers nach Nr. 5). ${ }^{104}$ Man kann also danach nur von einem eingeschränkten oder unechten Vetorecht sprechen. Was aber genau bedeutet dies? Da die Auslegung nicht im Widerspruch zur Verfassung stehen darf, ist der Blick auf das Grundgesetz zu richten.

Das Grundschulkind ist, wie oben ausgeführt, Träger der Menschenwürde (Art. 1 Abs. 1 GG) sowie des Rechts auf körperliche Unversehrtheit (Art. 2 Abs. 2 GG). Es kann bereits bewusst einen eigenen Willen bilden. Jedoch ist unklar, inwieweit dieser Wille rechtlich maßgeblich sein kann, da er ggf. nicht auf der erforderlichen Einsichtsfähigkeit beruht.

\section{aa) Menschenwürde}

Dass das Grundschulkind definitionsgemäß noch nicht in der Lage ist, „Wesen, Bedeutung und Tragweite der Entnahme zu erkennen“ und seinen „Willen hiernach

99 Listl, (Fn. 52) $\$ 40$ AMG Rn. 36.

100 Listl, (Fn. 52) $\$ 40$ AMG Rn. 36.

101 Vgl. bereits BGHSt 16, 309 (312).

102 Kern, arzneimittelrechtliche Forschung (Fn. 3), S. 147 f.

103 BT-Drs. 15/2849, S. 61: „Beachten bedeutet, dass der Wille des Minderjährigen in dieser Fallgestaltung dem etwa abweichenden Willen des gesetzlichen Vertreters vorgehen würde, d. h. dass die Teilnahme des Minderjährigen an der klinischen Prüfung nicht zulässig ist."

104 Roth (Fn. 93), $\$ 8$ a TPG Rn. 10. Roth weist darüber hinaus darauf hin, dass aus einer Ablehnung in der Regel darauf geschlossen werden könne, dass die Einwilligung in den Eingriff nicht dem Wohl des Kindes entspricht. 
auszurichten“ (sonst wäre es nach der hier eingeführten Terminologie ein „Teenager"), bedeutet nicht, dass es über gar keine Einsichtsfähigkeit und über einen nur völlig unbeachtlichen Willen verfügt. Aus verfassungsrechtlicher Sicht muss die Beachtung des Willens entsprechend der Einsichtsfähigkeit des Kindes garantiert werden.

Dazu bedarf es zweierlei Anforderungen, die mindestens im Wege einer verfassungskonformen Auslegung (besser noch einer expliziten Regelung) sichergestellt werden müssten: Erstens muss der aufklärende Arzt objektiv sein (oder sich als „Anwalt“ des Spenderkindes verstehen) und zweitens muss einer „objektiven Instanz“ die Pflicht auferlegt werden, die Reaktion des Kindes zu werten und den so ermittelten Willen in die Spendeentscheidung mit einzubeziehen.

Da das Grundschulkind noch nicht in der Lage ist, alles zu überblicken und seine eigene $\mathrm{Zukunft}^{105} \mathrm{zu}$ antizipieren, ist es vertretbar, den Kindeswillen nicht allein ausschlaggebend sein zu lassen, sondern in Beziehung zum (objektiven) Kindeswohl zu setzen. Dies muss auch vor dem Hintergrund gelten, dass der „geäußerte Wille“ nicht notwendigerweise auch dem „wahren Willen“ des Kindes entsprechen muss ${ }^{106}$ und daher auch eine Auslegung des Willens erforderlich ist.

In jedem Falle aber muss die Reaktion auch des Grundschulkindes ernstgenommen und interpretiert werden. Im Falle einer ablehnenden Haltung darf eine Knochenmarkspende nur im Ausnahmefall vorgenommen werden, nämlich dann, wenn überwiegend wahrscheinlich ist, dass diese dem (psychischen) Wohl des Spenderkindes dient. Auch eine Ablehnung aufgrund einer unspezifischen Angst vor dem Eingriff ist ernst zu nehmen und darf nicht einfach übergangen werden.

Da sich das Grundschulkind gerade durch seine Teileinsichtsfähigkeit auszeichnet, muss besonders darüber gewacht werden, dass die stellvertretende Spendeentscheidung wirklich auf einem Mangel an Selbstbestimmungsfähigkeit bzw. Einsichtsfähigkeit beruht und nicht auf einer überdehnten fremdbestimmten Fürsorge, die die Individualität und Einsichtsfähigkeit des Kindes übergeht. Ansonsten liegt ein Verstoß gegen Art. 1 Abs. 1 GG vor. ${ }^{107}$

\section{bb) Recht auf körperliche Unversehrtheit und allgemeine Handlungsfreiheit}

Das Recht auf körperliche Unversehrtheit (Art. 2 Abs. 2 GG) kann den Staat dazu verpflichten, das Kind vor übergriffigem Handeln Dritter zu bewahren (Schutzpflicht). Es genügt hier die Grundrechtsträgerschaft des Minderjährigen, auf eine Grundrechtsausübungsfähigkeit kommt es nicht an, da es sich nicht um ein Grund-

105 Vgl. zur Frage, ob und inwieweit man eine Maßnahme befürworten kann, die sich erst in der Zukunft positiv auswirkt, die Diskussion von Brumlik und Schleiermacher bei Zitelmann, Kindeswohl und Kindeswille (Fn. 21), S. 83.

106 Vgl. Zitelmann, Kindeswohl und Kindeswille (Fn. 21), S. 35.

107 Vgl. auch Bauer (Fn. 62), Rn. 27. 
recht handelt, das bestimmte geistige Fähigkeiten voraussetzt. ${ }^{108}$ In Frage steht hier, inwieweit es legitim ist, dass ein staatliches Gesetz die Eltern ermächtigt, das Kind zu einer primär fremdnützigen Spende zu zwingen. Hier kann an die unter oben zur Menschenwürde genannten Kriterien angeknüpft werden: Der Wille des Kindes darf nur unter den dort genannten engen Voraussetzungen übergangen werden.

\section{cc) Verfassungskonforme Auslegung des „eingeschränkten Vetorechts“ und Vorschlag einer Absicherung durch Kommissionsentscheidung}

Daraus folgt, dass $₫ 8$ a Nr. 4 S. 4 TPG verfassungskonform so zu interpretieren ist, dass erstens auch eine unspezifische Angstreaktion, ${ }^{109}$ die sich klar gegen den Eingriff richtet, beachtlich ist. ${ }^{110}$ Zweitens darf eine Ablehnung nur dann übergangen werden, wenn der Eingriff dem Kindeswohl des Spenderkindes dient, nicht aber bereits wegen einer Kosten-Nutzen-Abwägung im Hinblick auf den Gewinn für das Empfängerkind und die Risiken für das Spenderkind. Eine Sozialpflichtigkeit, ${ }^{111}$ die dahin reicht, dass sich jemand einer Knochenmarkspende auch gegen seinen Willen unterziehen muss, wäre mit dem Grundgesetz nicht zu vereinbaren. Zu Recht wird daher in der Literatur gefordert, dass im Zweifel der Ablehnung des Kindes Folge zu leisten ist. $^{112}$

Der offene Wortlaut und die verbleibenden Auslegungszweifel sind gerade vor dem im Strafrecht ${ }^{113}$ geltenden Bestimmtheitsgrundsatz ${ }^{114}$ nicht hinnehmbar. Vor diesem Hintergrund erschiene es sinnvoll, dass der Gesetzgeber, wie unter D. II näher ausgeführt, die Zulässigkeit einer Spende eines Babys und Grundschulkindes von einer positiven Entscheidung einer interdisziplinären Kommission abhängig macht. Für den Fall des ablehnenden Grundschulkindes könnte auf diese Weise auch geklärt werden, ob ausnahmsweise (!) doch eine Spende zulässig ist, weil diese nach aller Wahrscheinlichkeit auf längere Sicht dem (psychischen) Wohl des Spenderkindes dienlich wäre. Ebenso könnte durch den Einbezug einer Kommission einer Auslegung

108 Vgl. P. Kunig, in: I. v. Münch/P. Kunig (Hrsg.), Grundgesetz-Kommentar, Bd. 1, 6. Aufl., München 2012, Art. 2 Rn. 6; Roth, Die Grundrechte Minderjähriger (Fn. 21), S. 48. Das von Roth zu Art. 2 Abs. 1 GG Gesagte kann hier im „Erst-Recht-Schluss“ übertragen werden, weil der Schutz vor körperlichen Eingriffen umso weniger eine geistige Reife erfordert.

109 Vgl. zu den Ängsten Roell, Geschwister (Fn. 18), S. 307.

110 Vgl. Schmidt-Recla, Kontraindikation (Fn. 7), S. 569. Vgl. auch aus den Gesetzesmaterialien zu $\$ 40$ Abs. 4 Nr. 3 AMG: BT-Drs. 15/ 2849, S. 61: „Eine Ablehnung auf sonstige Weise kann insbesondere durch nichtsprachliche Zeichen der Furcht oder des Schreckens zum Ausdruck kommen, sofern dies dauerhaft die Haltung des Minderjährigen zum Ausdruck bringt und unmissverständlich geschieht. “

111 Eine Sozialpflichtigkeit ablehnend auch A. Loose, Strafrechtliche Grenzen ärztlicher Behandlung und Forschung, Berlin 2003, S. 187 ff; Mehlitz, Die rechtliche Zulässigkeit placebokontrollierter klinischer Prüfungen (Fn. 64), S.176; M. Wunder, Unrecht durch Ungleichbehandlung oder Gleichbehandlung im Unrecht? JZ 2001, 344 (als Erwiderung auf E. Picker, Menschenrettung durch Menschennutzung? JZ 2000, 693).

112 Roth (Fn. 93), $\int 8$ a TPG Rn. 10 („muss auch hier im Zweifel von einer Ablehnung ausgegangen werden").

113 Vgl. $\$ 19$ Abs. 1 Nr. 1 TPG.

$114 \mathbb{\$} 1$ StGB; Art. 103 Abs. 2 GG. 
Raum gegeben werden, die die Ausübung des eingeschränkten Vetorechts des Grundschulkindes auch direkt gegenüber dem Arzt zulässt, wenn der Arzt die Möglichkeit erhält, für den Fall eines Konflikts mit der elterlichen Einwilligung die Kommission anzurufen.

\section{dd) Ausübungsfrist}

Besonders problematisch ist allerdings, dass sich eine Ablehnung eines Eingriffs auch und gerade erst in letzter Sekunde manifestieren kann, nämlich wenn tatsächlich „Hand an das Spenderkind“ gelegt wird. Würde man ein so spätes Ablehnungsrecht noch zulassen, wäre dies beinahe das Todesurteil für das Empfängerkind, wenn dessen Konditionierung schon im Gange ist. ${ }^{115}$

\ 8 a Nr. 4 S. 4 TPG bestimmt nicht ausdrücklich, bis zu welchem Zeitpunkt eine Ablehnung zu beachten ist, auch wenn sie textlich direkt nach der Arztaufklärung verankert wurde und möglicherweise in diesem Zusammenhang gedacht war. Jedoch wäre die Vorstellung, ein Kind, welches selbst nicht aktiv zugestimmt hat, sondern über seine Eltern „fremdbestimmt“ wurde, zwangsweise einer OP zu unterziehen, obwohl es sich gegen den Eingriff massiv wehrt, unerträglich. Mindestens ebenso unerträglich wäre indes auch die Vorstellung, dem Willen des Spenderkindes Raum zu geben und dem Empfängerkind, dessen Konditionierung bereits begonnen hat, den Spender zu entziehen.

Rechtlich müsste dieses (eingeschränkte) Vetorecht des Kindes analog der Widerrufsmöglichkeit der Einwilligung zur Spende zu behandeln sein. $\int 8$ a TPG regelt keine spezifische Widerrufsfrist, so dass sich diese nach $\$ 8$ Abs. 2 S. 6 TPG ${ }^{116}$ bemisst. Eine zeitliche Grenze der Widerrufsfrist ist auch hier nicht geregelt. Da $\mathbb{S} 8$ Abs. 2 S. 7 TPG nur den S. 3 für die Knochenmarkspende ausnimmt, gilt die unbegrenzte Widerrufsfrist an sich auch für Knochenmarkspenden. ${ }^{117}$

Bei einem Widerruf Volljähriger nach $\mathbb{\int} 8$ Abs. 2 S. 6 TPG sprechen jedoch gute Gründe dafür, die Widerrufsmöglichkeit auf den Zeitpunkt des Beginns der Konditionierung zu begrenzen, da ein Volljähriger sich ein venire contra factum propri$u m$ vorwerfen lassen müsste, wenn er seine Einwilligungserklärung widerruft, nachdem der Empfänger im Vertrauen auf diese Einwilligung sein Leben in einen Grenzzustand verbracht hat.

115 Vgl. BÄK, Richtlinie für die allogene Knochenmarktransplantation mit nichtverwandten Spendern, Deutsches Ärzteblatt (91) 1994, A-761 (A-765).

116 Esser (Fn. 44), Rn. 32, 35, 107 (hier nur für Organspenden, da noch vor Verabschiedung des Gewebegesetzes).

117 In der allerdings noch vor dem Erlass des Gewebegesetzes von der BÄK verabschiedeten Richtlinie (Fn. 1) heißt es unter 3.4.: „Der Spender ist in geeigneter Weise darauf hinzuweisen, daß ein Rücktritt von der Knochenmarkspende zu einem späteren Zeitpunkt, insbesondere nachdem die unmittelbare Vorbereitung des Empfängers für die Knochenmarktransplantation begonnen hat, eine unmittelbare Lebensgefährdung des Empfängers bedeuten würde." Vgl. auch Schmidt-Recla, Kontraindikation (Fn. 7), S. 569. 
Für einen Grundschüler, der zuvor gar nicht selbst eingewilligt hat, ist die Argumentation der Rechtsmissbräuchlichkeit und des Vertrauensschutzes des Empfängers schon weniger evident. Eine eindeutige verfassungskonforme Auslegung dergestalt, dass dem Staat hier eine Schutzpflicht des Lebens des Empfängers auf Kosten der körperlichen Unversehrtheit des minderjährigen Spenders obliegt, wäre jedenfalls rechtlich nicht unproblematisch. Es eröffnet sich hier eine echte Dilemmasituation, die noch einmal vor Augen führt, wie brisant eine rechtliche Regelung der Spende eines „Grundschulkindes“ ist.

Vor dem Hintergrund des „irreversibel tragischen Vertrauens“, dass das Empfängerkind investiert hat und der Tatsache, dass normalerweise keine körperlichen Langzeitfolgen für das Spenderkind drohen, könnte man wohl aber auch hier argumentieren, dass das Grundschulkind, das ja zuvor durch den Arzt aufgeklärt wurde, zu einem so späten Zeitpunkt nicht mehr sein „eingeschränktes Vetorecht“ ausüben darf und die Eltern ihre Einwilligung nicht mehr widerrufen dürfen, mindestens dann, wenn der Arzt darauf hingewiesen hat, was ein späterer Rückzug für das Empfängerkind bedeutet.

\section{c) Bejahung der Spende durch das Grundschulkind}

Lehnt das Grundschulkind die Knochenmarkentnahme nicht ab, so reduzieren sich die Anforderungen an das „Kindeswohl“. Der Wille des teileinsichtsfähigen Grundschulkindes ist also auch hier zu beachten. Daher scheint es vertretbar, eine Knochenmarkspende zuzulassen, wenn die Prognose dahin ausfällt, dass der Eingriff dem Kind nützen könnte, in einer Gesamtbilanz jedenfalls aber nicht schaden würde. Willigen die Eltern eines spendewilligen Kindes nicht ein, darf das Grundschulkind nach $\mathbb{8}$ a Nr. 4 S. 1 TPG nicht spenden. Hier dürfen die Eltern die Autonomie des Grundschulkindes entgegen seinem Willen zulässig nach Art. 6 Abs. 2 GG einschränken, weil gerade Zweifel daran bestehen, dass es im Hinblick auf die Spendeentscheidung einsichtsfähig ist.

\section{Fallgruppe: Spendewillige Eltern und zustimmender Teenager}

Im Rahmen dieser Fallgruppe ist zunächst besonders sorgfältig zu klären, wann ein Kind überhaupt in diese Fallgruppe („Teenager“) einzustufen ist, wann es also in der Lage ist, Wesen, Bedeutung und Tragweite der Entnahme zu erkennen und seinen Willen danach auszurichten. Immerhin hängt von dieser Einschätzung ab, ob auch die Einwilligung des Minderjährigen erforderlich ist ( $\$ 8$ a Nr. 5 TPG). In der Gesetzesbegründung heißt es, dass diese Einsichtsfähigkeit „,in der Regel vom vollendeten 16. Lebensjahr an gegeben sein" kann. ${ }^{118}$ Eine starre Altersgrenze ist allerdings strikt

118 BT-Drs. 16/3146, S. 29 (rechte Spalte), vgl. auch BT-Drs. 15/2109, S. 31 (linke Spalte). In der Literatur werden schon jüngere Kinder für regelmäßig einsichtsfähig befunden: Golbs, Das Vetorecht (Fn. 4), S. 92; Listl, (Fn. 52), Rn. 36; Roth (Fn. 93), \$ 8 a TPG Rn. 11. 
abzulehnen. ${ }^{119}$ Vielmehr sind die Anforderungen, die an die Einsichtsfähigkeit gestellt werden, in Abhängigkeit von der Schwere des Eingriffs zu bestimmen. ${ }^{120}$

Im Falle eines spendewilligen Teenagers und einwilligender Eltern scheint es auf den ersten Blick kein Problem zu geben. Auf den zweiten Blick aber kann sich auch diese Situation zu Lasten des Teenagers auswirken. Gerade wenn die Eltern zum Ausdruck bringen, dass sie eine Spende wünschen oder wenn der aufklärende Arzt betont, dass nur noch er, der Teenager, das Geschwisterkind retten könne, kann sich eine Drucksituation ergeben, in der eine freie, autonome Willensentscheidung nicht mehr möglich ist. Ist der äußere Druck sehr hoch und sind die Erwartungen seitens der Familie klar formuliert, spricht sogar eine Vermutung dafür, dass eine autonome freiwillige Willensentscheidung des Kindes nicht mehr möglich ist. ${ }^{121}$

\section{Fallgruppe: Ablehnende Eltern und spendewilliger Teenager}

Lehnen die Eltern eine Spende ab, darf auch der (einsichtsfähige) Teenager nicht spenden. Wie ist diese Bevormundung des Teenagers verfassungsrechtlich zu beurteilen? Nimmt man - wie hier vertreten - an, dass das fremdbestimmte Kindeswohlkriterium nur so lange Bedeutung hat, wie davon auszugehen ist, dass keine (volle) Einsichtsfähigkeit gegeben ist, so drängt sich die Frage auf, ob das „Mitbestimmungsrecht" der Eltern verfassungsrechtlich legitim ist.

\section{a) Art. 6 Abs. 2 GG}

Das Elternrecht nach Art. 6 Abs. 2 GG tritt immer mehr zurück, je mehr Einsichtsfähigkeit das Kind erlangt. ${ }^{122} \$ 8$ a TPG weist demgegenüber den Eltern eine Entscheidungsbefugnis in einem Bereich zu, für den der Minderjährige als einsichtsfähig eingestuft wurde. Damit geht er über das verfassungsrechtlich garantierte Elternrecht hinaus. Elternrecht und Grundrechte des Kindes müssen nicht gegeneinander abgewogen werden, sondern das Elternrecht tritt zurück, sobald das Kind seine Grundrechte aufgrund seiner Einsichtsfähigkeit allein ausüben kann. ${ }^{123}$

\section{b) Grundrechte des Teenagers}

Das Grundrecht auf körperliche Unversehrtheit (Art. 2 Abs. 2 GG) vermittelt kein Recht zur Selbstverletzung. Das gesetzlich vorgesehene Mitbestimmungsrecht der Eltern, das gesetzlich am Wohl des Minderjährigen ausgerichtet ist, greift aber in die allgemeine Handlungsfreiheit (Art. 2 Abs. 1 GG) des (einsichtsfähigen) Teenagers

119 Gegen eine starre Altersgrenze für die Bestimmung der Einwilligungsfähigkeit: Golbs, Das Vetorecht (Fn. 4), S. 90 ff.: Alter kann nur groben Anhaltspunkt bieten.

120 Ch. Kopetzki/A. Leischner, Juristische Stellungnahme zur Nierentransplantation unter eineiigen Zwillingen im Alter von 13 Jahren, Recht der Medizin (RdM) 2009, S. 12 (für die österreichische Rechtslage).

121 Vgl. Fn. 18.

122 Nachweise in Fn. 87. Gegen eine Mitbestimmung durch die Eltern: Schmidt-Recla, Kontraindikation und Kindeswohl (Fn. 7), S. 569 f. S. auch Kern, Fremdbestimmung (Fn. 28), S. 755.

123 Vgl. oben B. 
ein. Eine Rechtfertigung für diesen Eingriff könnte darin begründet sein, dass die Knochenmarkentnahme den Minderjährigen körperlich wie psychisch belasten kann. Geht man davon aus, dass der Teenager einsichtsfähig ist, wäre ein „Schutz vor sich selbst" aber nur dann gerechtfertigt, wenn die spezifische Stellung als minderjähriges Kind in der Familienkonstellation eine freiwillige Entscheidung und damit Sorge für sich selbst unmöglich machen würde. ${ }^{124}$ Gerade bei Eltern, die die Spende ablehnen, wäre aber dies nicht zu befürchten. Ebenso handelt es sich bei der Knochenmarkspende nicht um einen risikoreichen oder irreversiblen Eingriff wie bei einer Lebendorganspende, bei dem der Minderjährigenschutz so hoch gehängt werden müsste, um auszuschließen, dass aufgrund einer Fehleinschätzung der Einsichtsfähigkeit des Teenagers unumkehrbare Ergebnisse geschaffen werden. Vielmehr ist die Knochenmarkentnahme in der Regel nur mit dem Operationsrisiko und kurzfristigen reversiblen Gesundheitsbeeinträchtigungen verbunden, so dass der einsichtsfähige Teenager hierüber selbst aufgrund seiner allgemeinen Handlungsfreiheit entscheiden können muss.

Man könnte überdies erwägen, die Entscheidung zur Stammzellspende auch im allgemeinen Persönlichkeitsrecht (Art. 2 Abs. 1 i.V.m. Art. 1 Abs. 1 GG) verankert zu sehen. Das allgemeine Persönlichkeitsrecht wurde in Fallgruppen vom BVerfG entwickelt, die diesen Fall zwar nicht direkt erfassen, einer Erweiterung aber zugänglich sind. So könnte man erwägen, die Bestimmung über den eigenen Körper als neue Fallgruppe des allgemeinen Persönlichkeitsrechts anzusehen. Jedoch geht es hier nicht um den Schutz des Körpers (wie ihn bereits Art. 2 Abs. 2 GG vermittelt), sondern um die Verfügung über den Körper zu seinem Nachteil. Würde man ein solches Recht unter den grundrechtlichen Schutz des Art. 2 Abs. 1 i.V.m. Art. 1 Abs. 1 GG stellen, so hätte man gewissermaßen ein Recht auf Selbstverstümmelung etabliert. Eine solche Fallgruppe unter dem allgemeinen Persönlichkeitsrecht als explizites Grundrecht zu garantieren, widerspräche der vielfach auf Schutz des Menschen angelegten Rechtsordnung. Es erscheint daher angemessener, die Autonomie, sich selbst Schaden zuzufügen, lediglich über die einer Einschränkung leichter zugänglichen allgemeinen Handlungsfreiheit zu verbürgen.

Überdies ist zu differenzieren zwischen einer eigenhändig vorgenommenen Selbstverletzung und einer solchen durch fremde Hand (im Falle der Knochenmarkspende durch den Arzt). Auch andernorts geht der Gesetzgeber davon aus, dass die „Selbstverletzung auf Verlangen “ nicht als Ausdruck des Persönlichkeitsrechts geschützt ist

124 Vgl. hier auch die Grundsätze aus dem Betreuungsrecht, D. Schwab, in: F.J. Säcker/R. Rixecker (Hrsg.)/D. Schwab (Red.), Münchener Kommentar zum BGB, Bd. 8, 6. Aufl. München 2012, $\int 1896$ Rn. 25 (Rechtsprechungssicht); S. Sonnenfeld, Das 2. BtÄndG, FamRZ 2005, S. 941; BVerfG FamRZ 2010, S. 1624 (1625); BayOblG, FamRZ 2003 (962). 


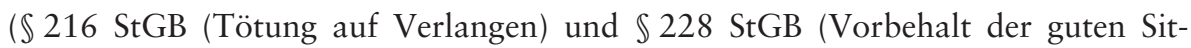
ten)). ${ }^{125}$

Die restriktive Regelung der Lebendorganspende durch das TPG wurde bereits vom BVerfG bestätigt, ${ }^{126}$ indem es das gesetzgeberische Anliegen akzeptierte, den Spender vor sich selbst zu schützen. Auch das BVerfG prüfte in dieser Entscheidung zur Lebendorganspende in Bezug auf den Wunschspender lediglich eine mögliche Verletzung von Art. 2 Abs. 1 GG, nicht aber von Art. 2 Abs. 1 i.V.m. Art. 1 Abs. 1 GG. ${ }^{127}$ Dies ist gerade vor dem Hintergrund sinnvoll, dass die Einschränkung des allgemeinen Persönlichkeitsrechts (im Gegensatz zur allgemeinen Handlungsfreiheit) gesteigerten Anforderungen unterliegt und dass dieses Recht bei einer zu weiten Fassung letztlich auf der Rechtfertigungsebene eine gravierende Lockerung erfahren müsste und dadurch am Ende an Schlagkraft für diejenigen Fälle verlieren würde, die mehr Schutz als nur über Art. 2 Abs. 1 GG erfordern. Die Entscheidung zur Stammzellspende wird bereits über die allgemeine Handlungsfreiheit (Art. 2 Abs. 1 GG) hinreichend geschützt und stellt nach hier vertretener Meinung keinen Sachverhalt dar, für den das allgemeine Persönlichkeitsrecht bemüht werden müsste.

Aus der staatlichen Schutzpflicht der körperlichen Unversehrtheit (Art. 2 Abs. 2 GG) kann allerdings in Begrenzung der Ausübung des Grundrechts der allgemeinen Handlungsfreiheit (Art. 2 Abs. 1 GG) abgeleitet werden, dass besonders hohe Anforderungen an die Feststellung der Einsichtsfähigkeit nach $\$ 8$ a Nr. 5 TPG zu stellen sind. Angemessen wäre etwa eine Entscheidung über die Einsichtsfähigkeit durch eine mit einem Psychologen, Sozialarbeiter und Arzt besetzte Kommission. Als allgemeine Kriterien für die Einsichtsfähigkeit gelten die geistige und sittliche Reife, die es dem Minderjährigen ermöglicht, die Tragweite des medizinischen Eingriffs zu ermessen. ${ }^{128}$

c) Fazit

Ein grundsätzlicher Ausschluss der freiwilligen Eigenentscheidung zur Stammzellspende verstößt gegen die allgemeine Handlungsfreiheit (Art. 2 Abs. 1 GG) des Teen-

125 Vgl. auch D. Norba, Rechtsfragen der Transplantationsmedizin aus deutscher und europäischer Sicht, Berlin 2009, S. $209 \mathrm{f}$.

126 BVerfG, Nichtannahmebeschluss, NJW 1999, S. 3399. Kritik an diesem Nichtannahmebeschluss durch: Th. Gutmann, Gesetzgeberischer Paternalismus ohne Grenzen? Zum Beschluß des Bundesverfassungsgerichts zur Lebendspende von Organen, NJW 1999, S. 3387; B. Seidenrath, Zur Verfassungsmäßigkeit der Regelungen über die Lebendspende bei der Organtransplantation, MedR 2000, S. 33. Gewisse Bestätigung dieses Beschlusses durch BVerfG, Nichtannahmebeschluss (Sonnenstudio-Verbot für Minderjährige), NJW 2012, S. 1062: Orientierungssatz 2 a: „Es ist grundsätzlich ein legitimes Gemeinwohlanliegen, Menschen davor zu bewahren, sich selbst leichtfertig einen größeren persönlichen Schaden zuzufügen."

127 Ebenso nur eine Prüfung von Art. 2 Abs. 1 GG bei B. Holznagel, Aktuelle verfassungsrechtliche Fragen der Transplantationsmedizin, DVBl 2001, S. 1629 (1634).

128 H. H. Lesch, Die strafrechtliche Einwilligung beim HIV-Antikörpertest an Minderjährigen, NJW 1989, S. 2309. 
agers. Dieses Ergebnis entspricht auch der überwiegenden Meinung, die zur Einwilligungsfähigkeit Minderjähriger in die Verletzung höchstpersönlicher Rechtsgüter im Strafrecht vertreten wird. ${ }^{129}$ Dort wird in der Regel auf die natürliche Einsichts- und Urteilsfähigkeit des Minderjährigen abgestellt. Im zivilrechtlichen Schadensersatzrecht wird vielfach allerdings nur ein Vetorecht des Minderjährigen angenommen. ${ }^{130}$ Verfassungsrechtlich gesehen bedeutet die Einschränkung des einsichtsfähigen Minderjährigen im Hinblick auf einen Eingriff, dessen Risiko voraussichtlich nicht über das Operationsrisiko oder leichte und reversible Gesundheitsbeeinträchtigungen hinausreicht, jedoch eine unzulässige Einengung seiner Handlungsfreiheit. Wenn das TPG, wie hier vorgeschlagen, die Eigenentscheidung des Teenagers zur Spende zulassen und das Kindeswohlkriterium zurücktreten lassen würde, müsste gesetzlich noch die hier vorgenommene Risikobegrenzung normiert werden. Mit der Einschränkung auf leichte und reversible Gesundheitsbeeinträchtigungen ist das Risiko enger gefasst als bei $\mathbb{S} 8$ Abs. 1 Nr. 1 lit. c TPG für Volljährige, um den Minderjährigenschutz angemessen zu gestalten.

\section{Sicherung des Kindeswohls durch Ergänzungspflegschaft oder Kommission}

Schließlich ist zu diskutieren, wer die „Einwilligung“ und Beurteilung des Kindeswohls des Minderjährigen vornehmen darf, wenn die Eltern in einem Interessenskonflikt stehen. Nach derzeitiger Gesetzeslage sind die Eltern als gesetzliche Vertreter entscheidungsbefugt und ihre Entscheidung ist praktisch gerichtlich nicht überprüfbar. Zwar könnte das Familiengericht nach $\$ 1666$ BGB einschreiten, jedoch ist in der Praxis schon fraglich, wer das Gericht überhaupt informieren sollte. Eine regelmäßige Überprüfung einer Knochenmarkspendeentscheidung der Eltern nach $\$ 1666$ BGB wäre systemwidrig, weil hier nur im Falle des konkreten Verdachts der Kindeswohlgefährdung eine Prüfung eingeleitet wird, nicht aber von vornherein jeder Fall der stellvertretenden Entscheidung zur Spende einer externen Kontrolle unterliegen würde. Auch würde eine gerichtliche Prüfung nach $\$ 1666$ BGB die Eltern in

129 H.-H. Jeschek/Th. Weigend, Lehrbuch des Strafrechts, Allgemeiner Teil, 5. Aufl., Berlin 1996, S. 382; Überblick bei Lesch, HIV-Antikörpertest an Minderjährigen (Fn. 128), S. 2309 sowie Deutsche Gesellschaft für Gynäkologie und Geburtshilfe e.V., Arbeitsgemeinschaft Medizinrecht, Stellungnahme zu Rechtsfragen bei der Behandlung Minderjähriger, November 2011, http://www.dggg.de/filead$\mathrm{min} /$ public_docs/Leitlinien/4-1-1-minderjaehrige-2011.pdf., S. 3.

130 K.O. Bergmann, in: K.O. Bergmann/B. Pauge/H.D. Steinmeyer/M. Alberts (Hrsg.), Gesamtes Medizinrecht, 1. Aufl., Baden-Baden 2012, S. 132; H.-M. Pawlowski, Allgemeiner Teil des BGB, 7. Aufl., Heidelberg 2003, Rn.167.; J. Gernhuber/D. Coester-Waltjen, Familienrecht, 6. Aufl., München 2010, \$ 57 Rn. 79 (für mehr Entscheidungsfreiheit des Minderjährigen). BGHZ 29, 33 ff., wonach bei medizinischen Heileingriffen die Vorschriften des zivilrechtlichen Minderjährigenschutzes nicht anwendbar sind. Eine analoge Anwendung wurde ebenfalls verneint, weil im konkreten Fall (1) die Einholung der Einwilligung der Eltern nicht möglich war, (2) der Patient kurz vor der Volljährigkeit stand und (3) „der Kläger nach seiner geistigen Veranlagung und Entwicklung sowie seiner sittlichen Reife fähig war, die Erheblichkeit und möglichen Folgen der Operation zu ermessen “; BGH NJW 2007, S. 217 für ein Vetorecht bei „einem nur relativ indizierten Eingriff mit der Möglichkeit erheblicher Folgen für ihre künftige Lebensgestaltung “. 
eine Beklagtenposition versetzen und gewissermaßen stigmatisieren, insbesondere wenn tatsächlich eine gerichtliche Anordnung getroffen wird. Im Übrigen war das

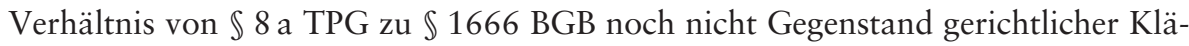
rung und ist auch von der Literatur noch nicht hinreichend erörtert worden. ${ }^{131}$

Zwei andere Wege sollen daher hier diskutiert werden:

\section{Ergänzungspflegschaft (\$ 1909 BGB)}

\1909 S. 1 BGB bestimmt:

„Wer unter elterlicher Sorge oder unter Vormundschaft steht, erhält für Angelegenheiten, an deren Besorgung die Eltern oder der Vormund verhindert sind, einen Pfleger. “

Für die Frage der Kindeswohlbestimmung und der Einwilligung in die Spende könnte also nach $\$ 1909$ BGB ein Ergänzungspfleger bestellt werden, der das nicht oder nicht voll einsichtsfähige Kind in dieser Angelegenheit vertritt. Sowohl die Bestimmung des Kindeswohls als auch die Vertretung des Kindes in Hinblick auf die Einwilligung zur Knochenmarkspende lägen dann beim Ergänzungspfleger. Diese Lösung würde jedoch die Eltern gänzlich aus der Entscheidungskompetenz verdrängen und diese nicht nur kontrollieren. Dies würde über das hier erforderliche Maß hinausgehen.

\section{Interdisziplinäre Kommission}

Eine zweite Möglichkeit wäre, dass das Kindeswohl durch eine interdisziplinäre Kommission bestimmt wird und erst nach einem positiven Votum die Eltern berechtigt sind, in Vertretung des Kindes die Einwilligung zur Spende abzugeben. Auf diese Weise würde den Eltern die Vertretungsmacht für die Spende nicht gänzlich aus der Hand genommen werden, sondern die Vertretungsmacht würde durch die Abhängigkeit von der Kommissionsentscheidung nur begrenzt werden. Den Weg über eine Ethikkommission ist der Gesetzgeber bereits in $\$ 40$ Abs. 1 S. 2 AMG gegangen. ${ }^{132}$ Ebenso gibt es bekanntlich die Lebendorganspende-Kommission nach $\mathbb{S} 8$ Abs. 3 TPG für die Organentnahme bei einem Lebenden, die einen Missbrauchsschutz kon-

131 Nach Gerdts, $\mathbb{\int} 8$ a (Fn. 12), ist $\mathbb{} 8$ a TPG lex specialis und $\mathbb{} 1666$ BGB daher nicht mehr anwendbar.

132 \40 Abs. 1 S. 2 AMG: „Die klinische Prüfung eines Arzneimittels bei Menschen darf vom Sponsor nur begonnen werden, wenn die zuständige Ethik-Kommission diese nach Maßgabe des $\mathbb{S} 42$ Abs. 1 zustimmend bewertet und die zuständige Bundesoberbehörde diese nach Maßgabe des $\mathbb{\int} 42$ Abs. 2 genehmigt hat." Auch die besonderen Bedürfnisse Minderjähriger hatte der Gesetzgeber in $\mathbb{S} 42$ Abs. 1 S. 5 AMG im Blick: „Sie hat Sachverständige beizuziehen oder Gutachten anzufordern, wenn es sich um eine klinische Prüfung bei Minderjährigen handelt und sie nicht über eigene Fachkenntnisse auf dem Gebiet der Kinderheilkunde, einschließlich ethischer und psychosozialer Fragen der Kinderheilkunde, verfügt oder wenn es sich um eine klinische Prüfung von xenogenen Arzneimitteln oder Gentherapeutika handelt." 
stituiert. ${ }^{133}$ An dieser Stelle ist auch das Erfordernis eines positiven Votums einer unabhängigen Ethikkommission im Falle der Blutstammzellspende (im Hinblick auf die Vorbehandlung der spendenden Person) besonders zu erwähnen ( $\mathbb{9}$ Abs. 1 S. 2 i.V.m. $\$ 8$ Abs. 2 Nr. 7 Transfusionsgesetz). Insofern wäre die Einbeziehung einer Kommission nicht gesetzesfremd, sondern beinahe schon systemimmanent.

Die Kommission könnte ggf. entsprechend dem Alters des Kindes unterschiedlich zusammengesetzt sein. Ein Team aus Kinderarzt, Familienpsychologe und Sozialarbeiter (und ggf. Jurist) könnte, nach Gesprächen mit dem Spenderkind und den Eltern befähigt sein, eine zuverlässigere Entscheidung darüber zu treffen, ob die Spende dem Wohl des Spenderkindes dient oder jedenfalls nicht schadet. Die Kommission könnte dabei selbstverständlich nicht gegen den Willen der Eltern entscheiden, deren $\mathrm{Zu}$ stimmung als gesetzliche Vertreter erforderlich bleibt. Ihr positives Votum wäre vielmehr ein kumulatives Erfordernis zur Einwilligung der Eltern, die Kommission hätte damit also nur eine Art „Vetorecht“. Die Kommissionsentscheidung über das Kindeswohl wäre nur solange erforderlich, wie die Eltern stellvertretend entscheiden dürfen.

Diese zweite Alternative (Kommission) ist der ersten (Ergänzungspflegschaft) vorzuziehen, weil die Eltern in die Entscheidung weiterhin einbezogen werden, es ihnen allerdings unmöglich gemacht würde, eine Entscheidung zu treffen, die das Wohl des Spenderkindes verletzt.

\section{E. Fazit}

Die Knochenmarkspende durch minderjährige Kinder an ihre Geschwister führt in ein Dilemma. Einerseits bedeutet sie Hoffnung auf Weiterleben für das kranke Geschwisterkind, also eine Dimension, der gegenüber die Nachteile für das Spenderkind gering erscheinen. Andererseits gilt rechtlich vor dem Hintergrund des Menschenwürdeschutzes das Prinzip, dass Gewebespenden nur auf freiwilliger Basis möglich sind und - bei Volljährigen - die Einwilligungsfähigkeit vorausgesetzt wird, um eine „Verzweckung“ einer Person zu verhindern.

Diese Grundsätze werden bei der Minderjährigenspende durchbrochen und sollen durch das „Kindeswohlkriterium“ aufgefangen werden. Der Begriff des Kindeswohls ist als Generalklausel auslegungs- und konkretisierungsbedürftig und bedarf einer

$133 \rrbracket 8$ Abs. 3 S. 2 ff. TPG: „Weitere Voraussetzung für die Entnahme von Organen bei einem Lebenden ist, dass die nach Landesrecht zuständige Kommission gutachtlich dazu Stellung genommen hat, ob begründete tatsächliche Anhaltspunkte dafür vorliegen, dass die Einwilligung in die Organspende nicht freiwillig erfolgt oder das Organ Gegenstand verbotenen Handeltreibens nach $\mathbb{} 17$ ist. Der Kommission muss ein Arzt, der weder an der Entnahme noch an der Übertragung von Organen beteiligt ist, noch Weisungen eines Arztes untersteht, der an solchen Maßnahmen beteiligt ist, eine Person mit der Befähigung zum Richteramt und eine in psychologischen Fragen erfahrene Person angehören. Das Nähere, insbesondere zur Zusammensetzung der Kommission, zum Verfahren und zur Finanzierung, wird durch Landesrecht bestimmt." 
verfassungskonformen Auslegung. In diesem Beitrag wird eine differenzierte Interpretation des Kindeswohls in Abhängigkeit der Einsichtsfähigkeit des Minderjährigen vorgeschlagen, um einerseits das Kindeswohlkriterium nicht zum bloßen Feigenblatt einer fremdbestimmten Spende verkommen zu lassen und andererseits Knochenmarkspenden nicht zur seltenen Ausnahme werden zu lassen.

Für das nicht einsichtsfähige Baby folgt aus der Korrelation von Kindeswohl und Einsichtsfähigkeit, dass eine Knochenmarkspende nur in den Ausnahmefällen zulässig ist, in denen eine überwiegende Wahrscheinlichkeit für den positiven Nutzen für das Spenderkind gegeben ist. Das Alter des nichteinsichtsfähigen Kindes (die Kategorie des Babys unterfällt noch einmal in verschiedene Entwicklungsstadien) ist hier zu berücksichtigen.

Beim teileinsichtsfähigen Grundschulkind richtet sich das Maß der Anforderungen an das Kindeswohl nach der Zustimmung bzw. Ablehnung des Minderjährigen. Stimmt das Grundschulkind zu, dürfte es ausreichen, wenn die Spende dem Kind jedenfalls nicht zum Schaden gereicht. Lehnt das Grundschulkind die Spende ab, ist die Spende unzulässig, es sei denn, dass die Spende (vs. einer Nichtspende) für das Grundschulkind in einer Gesamtbilanz mit hoher Wahrscheinlichkeit für sein psychisches Wohl positiv ist und dass - wie hier de lege ferenda gefordert - dieser wahrscheinlich positive Nutzen von einer interdisziplinären Kommission festgestellt wurde. Überdies sollte in allen Fällen der Spende eines Babys oder Grundschulkindes die Zulässigkeit zusätzlich von dem positiven Votum einer interdisziplinären Kommission abhängig gemacht werden, da sich die vertretungsberechtigten Eltern in einem unauflösbaren Interessenskonflikt zwischen Empfänger- und Spenderkind befinden.

Beim einsichtsfähigen Teenager sollte der Schutz vor einer Knochenmarktransplantation dagegen nicht mehr über das Kindeswohlkriterium erfolgen, sondern sein Schutz sollte über erhöhte Anforderungen zur Feststellung der Einsichtsfähigkeit gewährleistet werden. Vor seiner eigenen Entscheidung braucht der einsichtsfähige Teenager nicht geschützt zu werden, wenn gesetzlich sichergestellt ist, dass sich der Teenager durch die Spende nicht in eine Situation begeben kann, bei der er gesundheitlich irreversibel oder empfindlich beeinträchtigt würde. 